\title{
A NEW METHOD TO SEPARATE STAR-FORMING FROM AGN GALAXIES AT INTERMEDIATE REDSHIFT: THE SUBMILLIJANSKY RADIO POPULATION IN THE VLA-COSMOS SURVEY
}

\author{
V. Smolčíc, ${ }^{1,2,3}$ E. Schinnerer, ${ }^{1}$ M. Scodeggio, ${ }^{4}$ P. Franzetti, ${ }^{4}$ H. Aussel,${ }^{5,6}$ M. Bondi, ${ }^{7}$ M. Brusa, ${ }^{8}$ \\ C. L. Carilli, ${ }^{9}$ P. Capak ${ }^{3}$ S. Charlot,${ }^{6,10}$ P. Ciliegi, ${ }^{11}$ O. Ilbert,${ }^{5}$ Ž. Ivezić, ${ }^{12}$ K. Jahnke, ${ }^{1}$ \\ H. J. McCracken, ${ }^{6}$ M. Obrić, ${ }^{12}$ M. Salvato, ${ }^{3}$ D. B. Sanders, ${ }^{5}$ N. Scoville, ${ }^{3,13}$ \\ J. R. Trump,${ }^{14}$ C. Tremonti, ${ }^{15}$ L. Tasca, ${ }^{16}$ C. J. Walcher, ${ }^{6}$ and G. Zamorani ${ }^{11}$ \\ Received 2007 September 27; accepted 2008 February 28
}

\begin{abstract}
We explore the properties of the submillijansky radio population at $20 \mathrm{~cm}$ by applying a newly developed optical color-based method to separate star-forming (SF) from active galactic nucleus (AGN) galaxies at intermediate redshifts $(z \lesssim 1.3)$. Although optical rest-frame colors are used, our separation method is shown to be efficient and not biased against dusty starburst galaxies. This classification method has been calibrated and tested on a local radioselected optical sample. Given accurate multiband photometry and redshifts, it carries the potential to be generally applicable to any galaxy sample where SF and AGN galaxies are the two dominant populations. In order to quantify the properties of the submillijansky radio population, we have analyzed $\sim 2,400$ radio sources, detected at $20 \mathrm{~cm}$ in the VLA-COSMOS survey; $90 \%$ of these have submillijansky flux densities. We classify the objects into (1) star candidates, (2) quasi-stellar objects, (3) AGN, (4) SF, and (5) high-redshift ( $z>1.3$ ) galaxies. We find, for the composition of the submillijansky radio population, that SF galaxies are not the dominant population at submillijansky flux levels, as previously often assumed, but that they make up an approximately constant fraction of $30 \%-40 \%$ in the flux density range of $\sim 50 \mu \mathrm{Jy}$ to $0.7 \mathrm{mJy}$. In summary, based on the entire VLA-COSMOS radio population at $20 \mathrm{~cm}$, we find that the radio population at these flux densities is a mixture of roughly $30 \%-40 \%$ of SF and $50 \%-60 \%$ of AGN galaxies, with a minor contribution $(\sim 10 \%)$ of QSOs.
\end{abstract}

Subject headings: cosmology: observations — radio continuum: galaxies — surveys

Online material: color figures

\section{INTRODUCTION}

The most straightforward information that can be derived from extragalactic radio sky surveys are the radio source counts, which have been extensively studied in the last three decades (Condon 1984; Windhorst et al. 1985; Gruppioni et al. 1999;

\footnotetext{
${ }^{1}$ Max-Planck-Institut-für-Astronomie, Königstuhl 17, Heidelberg, D-69117, Germany.

${ }^{2}$ Fellow of the International Max Planck Research School for Astronomy and Cosmic Physics.

${ }^{3}$ California Institute of Technology, MC 105-24, 1200 East California Boulevard, Pasadena, CA 91125.

4 IASF Milano-INAF, Via Bassini 15, I-20133, Milan, Italy.

5 Institute for Astronomy, University of Hawaii, 2680 Woodlawn Drive, Honolulu, HI 96822.

${ }^{6}$ Institut d'Astrophysique de Paris, UMR 7095, 98 bis Boulevard Arago, 75014 Paris, France.

7 INAF-Istituto di Radioastronomia, via Gobetti 101, 40129 Bologna, Italy.

8 Max-Planck-Institut-für-Extraterrestrische Physik, D-85478 Garching, Germany.

${ }_{9}$ National Radio Astronomy Observatory, P.O. Box 0, Socorro, NM 878010387.

${ }^{10}$ Max-Planck-Institut-für-Astrophysik, Karl-Schwarzschild-Strasse 1, 85748 Garching, Germany.

${ }^{11}$ INAF-Osservatorio Astronomico di Bologna, via Ranzani 1, 40127, Bologna, Italy.

12 Department of Astronomy, University of Washington, Box 351580, Seattle, WA $98195-1580$.

${ }_{13}$ Visiting Astronomer, University of Hawaii, 2680 Woodlawn Drive, Honolulu, HI, 96822.

14 Steward Observatory, University of Arizona, 933 North Cherry Avenue, Tucson, AZ 85721.

${ }^{15}$ Hubble Fellow, University of Arizona, Steward Observatory, 933 North Cherry Avenue, Tucson, AZ 85721.

${ }_{16}$ Laboratoire d'Astrophysique de Marseille, BP 8, Traverse du Siphon, 13376 Marseille Cedex 12, France.
}

Seymour et al. 2004; Simpson et al. 2006). If space were Euclidian and there were no cosmic evolution of radio sources, then the differential source counts would follow a power law with an exponent of 2.5 (see, e.g., Peterson 1997). Hence, the observed slope (and the change of the slope) of the radio source counts in different flux density ranges provides an insight, although quite indirect, into the global properties of extragalactic radio sources and their cosmic evolution. Past studies have shown that at $1.4 \mathrm{GHz}$ flux densities above $\sim 100 \mathrm{mJy}$, the source counts are dominated by "radio-loud" active galactic nuclei (AGNs) with luminosities above the Fanaroff \& Riley (1974) break $\left(\sim 2 \times 10^{25} \mathrm{~W} \mathrm{~Hz}^{-1}\right.$; Willott et al. 2002). Decreasing from about $100 \mathrm{mJy}$ to $1 \mathrm{mJy}$, the source counts follow a power law (e.g., Windhorst et al. 1985) and are mostly made up of radio-loud objects with luminosities below the Fanaroff \& Riley break (FR class I sources). However, the differential source counts change their slope again, i.e., they flatten below $1 \mathrm{mJy}$, and these submillijansky radio sources have often been interpreted as a rising new population of objects which does not contribute significantly at higher flux densities (e.g., Condon 1984).

To date the exact composition of this faint radio population (hereafter population mix) is not well determined, and it is rather controversial. Windhorst et al. (1985) suggested that the majority of submillijansky radio sources are faint blue galaxies, presumably undergoing significant star formation. Optical spectroscopy, obtained by Benn et al. (1993) supported this idea, and the source counts at faint levels were successfully modeled with a population of intermediate-redshift star-forming galaxies (Huynh et al. 2005; Seymour et al. 2004). However, spectroscopic observations by Georgakakis et al. (1999) yielded a fraction of 50\% of starforming (SF) galaxies, while the spectroscopic results by Gruppioni et al. (1999) suggested that early-type galaxies were the dominant 
population at submillijansky levels. Furthermore, it was recently suggested and modeled that the flattening of the source counts may be caused by radio-quiet AGNs (radio-quiet quasars and type 2 AGNs), rather than SF galaxies (Jarvis \& Rawlings 2004); observations support this interpretation (Simpson et al. 2006). Based from the combination of optical and radio morphology as an identifier for AGN and SF galaxies, Fomalont et al. (2006) suggested that at most $40 \%$ of the submillijansky radio sources comprise AGNs, while Padovani et al. (2007) indicated that this fraction may be $60 \%-80 \%$ (the latter based their SF/AGN classification on a combination of optical morphologies, X-ray luminosities, and radio-to-optical flux density ratios of their radio sources). Recent optical spectroscopic data suggest that the fraction of AGN and SF galaxies in the submillijansky population is roughly equal (Cowie et al. 2004; Afonso et al. 2005).

Two main reasons exist for such discrepant results. First, the identification fraction of radio sources with optical counterparts, which is generally taken to be representative of the full radio population, spans a wide range in literature $(20 \%-90 \%)$ depending on the depth of both the available radio and the optical data, as well as the passband used (e.g., a larger fraction of radio sources are associated with near-infrared [NIR] than optical data; see $\S 3.2)$. Second, the methods that were used to separate AGN from SF galaxies have been very heterogeneous in the past, ranging from pure radio luminosity or morphology cuts through observed color properties to optical spectroscopy.

The two main populations of radio sources in deep radio surveys at $1.4 \mathrm{GHz}(20 \mathrm{~cm}$ ) are AGNs and SF galaxies (Condon 1984; Windhorst et al. 1985). At this frequency the radio emission predominantly arises from synchrotron emission powered either by accretion onto the central supermassive black hole (SMBH) or by supernovae remnants (e.g., Condon 1992; note that both mechanisms may be at work in a given galaxy). It was shown that radio properties such as the distributions of monochromatic luminosities of SF and AGN galaxies (Seyferts, LINERs) are comparable and overlapping (at least locally; e.g., Sadler et al. 1999). Hence, in order to disentangle SF and AGN galaxies in the radio regime, observations at other wavelengths are required.

Studies of extragalactic radio sources in the local universe $(z<0.3)$ have been invigorated due to the recent advent of panchromatic photometric and spectroscopic all-sky surveys, such as, e.g., the NRAO VLA Sky Survey (NVSS; Condon et al. 1998), Faint Images of the Radio Sky at Twenty cm (FIRST; Becker et al. 1995), Sloan Digital Sky Survey (SDSS; York et al. 2000), IRAS (Beichman et al. 1985), and Two Degree Fireld (2dF; Colless et al. 2001), which provide additional panchromatic photometric (e.g., Simpson et al. 2006) and/or optical-IR spectroscopic (e.g., Sadler et al. 1999; Best et al. 2005) observations. For example, the panchromatic properties of radio sources were studied in full detail (Ivezić et al. 2002; Obrić et al. 2006), as well as the environmental dependence of radio luminous AGN and SF galaxies (Best 2004) and their luminosity function (Sadler et al. 1999; Jackson \& Londish 2000; Chan et al. 2004; Best et al. 2005). Furthermore, radio emission as a star formation rate indicator was well calibrated using a local sample (Bell 2003) and compared to other star formation tracers (Hopkins et al. 2003).

However, it still remains to uncover the global properties of the intermediate-redshift $(z \lesssim 1.3)$ radio sources. For example, the cosmic star formation history of the universe (i.e., the global star formation rate per unit comoving volume as a function of redshift) was not determined with a high accuracy using radio data (see, e.g., Haarsma et al. 2000; Hopkins 2004), the radio luminosity function, and its evolution, for SF and AGN galaxies at $z>0.3$ is not determined with high precision (see Cowie et al.
2004; Afonso et al. 2005), and the exact composition of the submillijansky radio population is a matter of debate (Condon 1984; Windhorst et al. 1985; Gruppioni et al. 1999; Seymour et al. 2004; Jarvis \& Rawlings 2004; Simpson et al. 2006).

In this work and in a number of accompanying papers, we focus on these properties of radio sources using the $1.4 \mathrm{GHz}$ VLACOSMOS survey (Schinnerer et al. 2007). The main aim of the current paper is twofold. First, we develop a method based only on multiwavelength photometric data to efficiently separate SF from AGN galaxies in the VLA-COSMOS $20 \mathrm{~cm}$ survey. Second, we use this classification to derive the composition of the submillijansky radio population. In $\S 2$ we describe the COSMOS multiwavelength data, and in $\S 3$ we present the cross-correlation of the sources detected at $1.4 \mathrm{GHz}$ with catalogs at other wavelengths. In $\S 4$ we describe our source classification methodology and introduce our "rest-frame color-based classification method" (see below), which we calibrate and extensively test using a large well-characterized sample of local galaxies. We present the classification of the VLA-COSMOS $1.4 \mathrm{GHz}$ radio sources with identified optical counterparts in $\S 5$, and in $\S 6$ we compare our classification method with other classification schemes proposed in the literature. In $\S 7$ we study the population mix in the VLACOSMOS radio survey, based on the entire sample of VLACOSMOS radio sources. We summarize our results in $\S 8$.

Throughout the paper we report magnitudes in the AB system and assume the following cosmology: $H_{0}=70, \Omega_{M}=0.3, \Omega_{\Lambda}=$ 0.7 . We define the radio synchrotron spectrum as $F_{\nu} \propto \nu^{-\alpha}$, and assume $\alpha=0.8$ if not stated otherwise. Hereafter, we refer to our method of classifying the VLA-COSMOS radio sources into five subtypes of objects (star candidate, QSO, AGN, SF, high-z galaxy) as "classification method," and to our method of disentangling only the SF from AGN galaxies, based on rest-frame color properties, as "rest-frame color-based selection method."

\section{THE MULTIWAVELENGTH DATA SET}

In this section we describe the COSMOS multiwavelength data used for the work presented here.

\subsection{Radio Data}

The COSMOS field was observed at $1.4 \mathrm{GHz}(20 \mathrm{~cm})$ with the NRAO Very Large Array (VLA) in A- and C-configuration (VLACOSMOS Large Project; for details see Schinnerer et al. 2007). The final map, encompassing $2 \mathrm{deg}^{2}$, has a resolution of $1.5^{\prime \prime} \times 1.4^{\prime \prime}$ and a mean rms of $\sim 10.5$ (15) $\mu \mathrm{Jy} \mathrm{beam}^{-1}$ in the central 1 (2) deg ${ }^{2}$.

The VLA-COSMOS source catalog reports the peak and total (i.e., integrated) flux density for each object. For extended sources the total flux density is derived by integrating over the object's size (see Schinnerer et al. 2007 for details), while for unresolved sources it is set to be equal to the peak flux density. Bondi et al. (2007) have shown that bandwidth-smearing effects (i.e., chromatic aberration), combined with the pointing layout of the VLACOSMOS observations, systematically decrease the measured source's peak flux density to $\sim 80 \%$ of its true value, while the total flux density remains unaffected. Therefore, to correct for this, all peak flux densities in the catalog need to be increased by $25 \%$. However, such an effect further entails a necessary redefinition of the sources in the field considered to be unresolved (see Fig. 14 in Schinnerer et al. 2007 and Fig. 2 in Bondi et al. 2007). Therefore, to properly correct for bandwidth-smearing effects, we have reselected the unresolved sources following Bondi et al. (2007) and set their total flux densities to be 1.25 times their peak (respective integrated) flux densities. Throughout the paper we use the integrated flux density, corrected for bandwidth 
smearing where needed, as the representative flux density for each source.

In order to minimize the number of possible spurious radio sources $(\sim 50 \%$ below $5 \sigma)$, we select only objects from the catalog that were detected at a signal-to-noise ratio of $\geq 5$ and are located outside regions contaminated by sidelobes from nearby bright sources. This yields 2388 (out of 3643; i.e., 65\%) sources, 78 of which consist of multiple components.

\subsection{Near-Ultraviolet, Optical and Infrared Imaging Data}

The near-ultraviolet (NUV) to mid-infrared (MIR) imaging data and photometry for the COSMOS survey used here include data taken during 2003-2006 with ground-based (Subaru, Kitt Peak National Observatory [KPNO], Cerro Tololo Inter-American Observatory [CTIO], Canada-France-Hawaii Telescope [CFHT]) and space-based (Hubble Space Telescope [HST], Spitzer Space Telescope) telescopes, covering a wavelength range from $3500 \AA$ to $8 \mu \mathrm{m}$, described them in more detail below.

\subsubsection{Ground-based Data}

The data reduction of the COSMOS ground-based observations in 15 photometric bands ranging from NUV to NIR, and the generation of the photometric catalog, is presented in Capak et al. (2007) and Taniguchi et al. (2007). Here we make extensive use of the COSMOS photometric catalog. The photometric catalog was selected using the $0.6^{\prime \prime}$ resolution $i^{+}$image. However, the photometry was obtained from the point-spread function (PSF) matched images, which degrades the resolution to $\sim 1.8^{\prime \prime}$. The median $5 \sigma$ depths in $\mathrm{AB}$ magnitudes in the catalog for the $u^{*}$, $B_{J}, g^{+}, V_{J}, r^{+}, i^{+}, i^{*}, z^{+}$, and $K_{s}$ bands ${ }^{17}$ are 26.4, 27.3, 27.0, 26.6, $26.8,26.2,24,25.2$, and 21.6, respectively (see also Table 2 in Capak et al. 2007). It is noteworthy that the detection completeness of the catalog is above $87 \%$ for objects brighter than $i=26$.

\subsubsection{Space-based Data}

The HST ACS observations, which covered $1.8 \mathrm{deg}^{2}$ of the $2 \mathrm{deg}^{2}$ COSMOS field, are described in Scoville et al. (2007a) and Koekemoer et al. (2007). The F814W band imaging has a resolution of $0.09^{\prime \prime}$ and a $5 \sigma$ point-source sensitivity of $I_{\mathrm{AB}}=28.6$ (see also Capak et al. 2007). The ACS source catalog, which we use here, was constructed by Leauthaud et al. (2007) with special care given to the separation of point sources from extended objects.

The Spitzer observations of the COSMOS field in all seven bands $(3.6,4.5,5.8,8.0,24,70$, and $160 \mu \mathrm{m})$ are described in Sanders et al. (2007). The $3.6-8 \mu \mathrm{m}$ band catalog is available to full depth for the entire field. The resolution in the 3.6, 4.5, 5.8, and $8.0 \mu \mathrm{m}$ bands is $1.7^{\prime \prime}, 1.7^{\prime \prime}, 1.9^{\prime \prime}$, and $2^{\prime \prime}$, respectively. The catalog was generated using SExtractor on the four IRAC channels in dual mode, with the $3.6 \mu \mathrm{m}$ image as the detection image. The $5 \sigma$ depth for point sources at $3.6 \mu \mathrm{m}$ is $1 \mu \mathrm{Jy}$, corresponding to an AB magnitude of 23.9. In this work we also make use of the MIPS $24 \mu \mathrm{m}$ catalog obtained from the shallow observations of the entire COSMOS field during Cycle 2 of the S-COSMOS program (see Sanders et al. 2007 for details). The resolution and $5 \sigma$ depth of the catalog are $6^{\prime \prime}$ and $0.3 \mathrm{mJy}$, respectively. The latter corresponds to an $\mathrm{AB}$ magnitude of 17.7. For the purpose of this paper, we use only sources that were detected at $24 \mu \mathrm{m}$ at or above the $3 \sigma$ level corresponding to their local rms.

\footnotetext{
17 The plus sign superscript and the "J" subscript designate the Subaru filters, while the asterisk $\left(^{*}\right)$ stands for CFHT filters.
}

\subsection{X-Ray Data}

The full $2 \mathrm{deg}^{2}$ COSMOS field was observed with the $X M M$ Newton satellite EPIC camera for a total net integration time of $1.4 \mathrm{Ms}$ (for a description of the XMM-Newton COSMOS survey see Hasinger et al. 2007). The limiting flux density of the $X M M-N e w t o n$ COSMOS survey is $10^{-15} \mathrm{ergs} \mathrm{cm}^{-2} \mathrm{~s}^{-1}$ and $5 \times$ $10^{-15} \mathrm{ergs} \mathrm{cm}^{-2} \mathrm{~s}^{-1}$ in the soft $(0.5-2 \mathrm{keV})$ and hard $(2-10 \mathrm{keV})$ bands, respectively. The X-ray point-source detection is described in Cappelluti et al. (2007) and the optical identifications of the $\mathrm{X}$-ray sources for the first 12 observed XMM-Newton fields (over a total of $1.3 \mathrm{deg}^{2}$ ) are presented by Brusa et al. (2007). For the analysis presented here we use the catalog with 1865 optical counterparts of the XMM-Newton COSMOS point sources, drawn from the full $2 \mathrm{deg}^{2} X M M-N e w t o n$ mosaic (M. Brusa et al. 2008, in preparation).

\subsection{Photometric Redshifts}

The COSMOS photometric redshifts (O. Ilbert et al. 2008, in preparation) used here are based on an a large amount of deep multicolor data (P. Capak et al. 2008, in preparation; Taniguchi et al. 2007; T. Taniguchi et al. 2008, in preparation); six broad optical bands obtained at the Subaru telescope $\left(u^{+}, g^{+}, r^{+}, i^{+}, z^{+}\right)$ and 2 at CFHT $\left(u^{*}\right.$ and $\left.i^{*}\right), 8$ intermediate- and narrowband filters from the Subaru telescope (IA427, IA464, IA505, IA574, IA709, IA827, NB711, NB816), deep $K_{s}$-band data from the WIRCAM CFHT camera (H. J. McCracken et al. in preparation), and 3.6 and $4.5 \mu \mathrm{m}$ data from the Spitzer IRAC camera (Sanders et al. 2007). The photometric redshifts are estimated via a standard $\chi^{2}$ fitting procedure (Arnouts et al. 2002) using the code Le Phare ${ }^{18}$ written by S. Arnouts \& O. Ilbert. A major feature of this method is the calibration of the photometric redshifts using a spectroscopic sample of $\sim 1000$ bright galaxies $\left(I_{\mathrm{AB}}<22.5\right)$ obtained as part of the zCOSMOS survey (Lilly et al. 2007). We follow exactly the same calibration method as described in Ilbert et al. (2006): (1) a calibration of the photometric zero-points and (2) an optimization of the template spectral energy distributions (SEDs). This calibration method allows us to remove systematic offsets in the estimates of the photometric redshifts. A direct comparison between the photometric redshifts and the zCOSMOS spectroscopic redshifts shows that the photometric redshifts reach an accuracy of $\sigma[\Delta z /(1+z)] \sim 0.014$ at $i<22.5$. The fraction of catastrophic failures is less than $1 \%$ at $i<22.5$. Such an accuracy and robustness can be achieved thanks to both the intermediate bands and deep NIR photometric data. The photometric redshifts for the entire COSMOS population is described in full detail in O. Ilbert et al. (2008, in preparation). The galaxies in the sample used here are radio selected, i.e., they are not randomly drawn from the global COSMOS population. Therefore, in Figure 1 we show the comparison of the photometric and spectroscopic redshifts for a subsample of our VLA-COSMOS sources with available spectroscopy (see $\S 2.5)$. The accuracy is $\sigma[\Delta z /(1+z)] \sim$ 0.027 , which is somewhat lower than the accuracy for the full sample of COSMOS sources; however, it is still satisfactory.

As photometric redshift codes generally take into account only galaxy SED models, the photometric redshifts for broad-line AGNs are usually poorly estimated (not better than $\sigma[\Delta z /(1+z)] \sim 0.1$ with a large fraction of catastrophic outliers and no solutions found beyond $z \sim 1.1$ ), and alternative ways for their redshift computations must be applied. At the time of writing, no accurately estimated photometric redshifts for broad-line AGNs exist for the COSMOS project. The photometric redshifts for broad-line

18 See www.lam.oamp.fr/arnouts/LE_PHARE.html. 


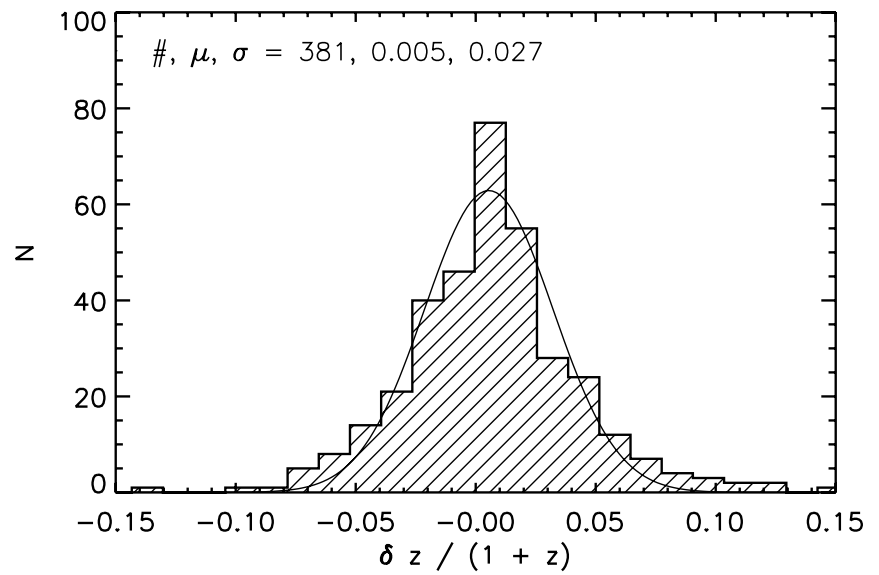

FIG. 1.-Comparison of the photometric and spectroscopic redshifts for the VLA-COSMOS radio sources with optical counterparts (see $\S 3.1$ ) for which spectroscopy is available. The distribution shown was limited to spectroscopic redshifts $\leq 1.3$, consistent with the redshift range used in this work. The number of sources, mean $(\mu)$, and standard deviation $(\sigma)$ of the distribution are indicated in the top left of the panel. Note the excellent accuracy of the photometric redshifts.

AGNs will be presented in a future publication (M. Salvato et al. 2008 , in preparation).

\subsection{Optical Spectroscopic Data}

The ongoing COSMOS optical spectroscopic surveys (Trump et al. 2007; Impey et al. 2007; Lilly et al. 2007) provide to date 657 spectra, with good redshift estimates, for objects in the VLACOSMOS 1.4 GHz radio sample described in $\S 2.1$. We augment this spectroscopic data set with available spectroscopic information for 65 galaxies from the SDSS DR4 "main" spectroscopic sample, 13 objects from the SDSS DR5 quasar catalog (Schneider et al. 2005), two sources from the $2 \mathrm{dF}$ survey, as well as for 27 objects taken with the MMT $6.5 \mathrm{~m}$ telescope, and presented by Prescott et al. (2006). Thus, a total of 764 spectra is available. However, as a number of sources were spectroscopically observed multiple times, we have spectroscopic information for 520 unique sources in our radio sample. Throughout the paper, we use the spectroscopic redshifts, where available. We also use this subset of radio sources with observed optical spectra as a control sample to verify the presented classification method.

\section{VLA-COSMOS $1.4 \mathrm{GHz}$ RADIO SOURCES AT OTHER WAVELENGTHS}

In this section we define the "matched radio source sample," a sample of radio sources with optical counterparts cross-correlated with the panchromatic COSMOS observations, as well as the "remaining radio source sample," both of which are used throughout the paper. First, we restrict the full VLA-COSMOS radio source sample to objects which have optical counterparts $(\S 3.1)$. Then we positionally match these objects with sources detected in the MIR ( $(3.2)$ and X-ray ( $§ 3.3)$ spectral ranges. In $\S 3.4$ we describe the remaining radio sources that are either without identified or with identified but flagged optical counterparts.

\subsection{Positional Matching of the COSMOS Radio and NUV/Optical/NIR Catalogs}

The VLA-COSMOS Large Project source catalog contains 2388 radio sources detected at a signal-to-noise ratio $\geq 5$ and outside sidelobe-contaminated regions (see $\S 2.1$ ). Of these, 78 consist of multiple components. For the purpose of this paper we match these radio sources with sources that have also been detected in

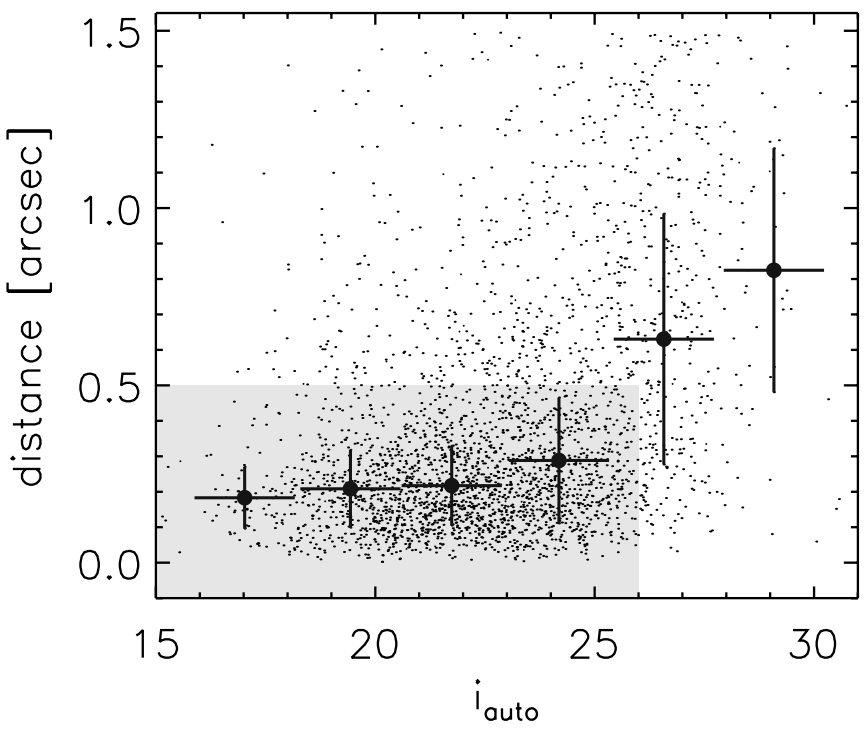

FIG. 2.-Distance as a function of $i$-band magnitude for radio sources in the VLA-COSMOS survey (with $\mathrm{S} / \mathrm{N} \geq 5$ ) positionally matched to the closest optical counterpart (dots). The large circles show the median distance for each magnitude bin (the width of the bin is indicated by horizontal lines) and the corresponding interquartile range (vertical lines). Note that the median distance is significantly larger beyond $i=26$, presumably introducing a significant number of false match associations. The shaded area indicates the allowed matching region, which is set by the matching radius $\left(0.5^{\prime \prime}\right)$ and the magnitude cutoff $(i=26)$ used to find secure optical counterparts for the radio sources in the sample (see text for details).

the optical regime and are reported in the COSMOS photometric catalog (Capak et al. 2007). In order to obtain a sample with reliable radio-optical counterparts, we positionally match the radio sources with only optical sources brighter than $i=26$. The reason for this is illustrated in Figure 2, where we show the distance between the radio sources and their nearest optical counterparts as a function of the $i$-band magnitude. As the median distance rapidly increases for $i>26$ (most probably introducing a significant number of false match associations) we apply a cut of $i=26$ to the NUV-NIR photometric catalog before matching the radio and optical catalogs.

Hence, to find the corresponding optical counterpart for each radio source (excluding multicomponent sources, which are separately addressed below), we search for the nearest optical neighbor within a radial distance of $0.5^{\prime \prime}$. The search radius was chosen in such a way that it balances a high completeness of true matches and a low false-match contamination rate. A cutoff of $0.5^{\prime \prime}$ essentially includes all true matches in the sample, with a false association rate (computed from the source density in the matched catalogs) of only $\sim 4 \%$. The high completeness and low contamination are due to the excellent astrometric accuracy of both the COSMOS radio and optical data. Our matching yielded 1749 radio sources with securely identified optical counterparts. However, $252(\sim 15 \%)$ of these are located in masked-out regions (i.e., around bright saturated stars) in the photometric catalog. Thus, their NUV-NIR photometry, as well as the photometric redshift computation has a significantly reduced accuracy. We exclude these objects from our main sample.

The multicomponent radio sources in the VLA-COSMOS survey consist of radio sources which could not be fitted using a single Gaussian function (see Schinnerer et al. 2007). The radio morphologies of such sources can be fairly complex (e.g., single or double lobed radio galaxies), and this makes it substantially more difficult to associate such radio sources with the appropriate optical counterparts (see, e.g., Ivezić et al. 2002; Best et al. 
2005a). In order to avoid any biases which may be caused using an automatic association procedure, the optical counterparts of the VLA-COSMOS multicomponent radio sources were determined visually. The $1.4 \mathrm{GHz}$ catalog contains 78 multicomponent sources detected at or above $5 \sigma$, and 65 were securely associated with an optical counterpart with $i<26$; however, four are located inside masked-out areas (around bright saturated stars) in the photometric catalog, and we therefore exclude them from the main sample.

In summary, the applied matching criteria yield $1814(\sim 76 \%$ of the 2388 radio sources with $\mathrm{S} / \mathrm{N} \geq 5$ ) radio sources with secure optical counterparts down to $i=26,65$ of which are multiple component sources. The most accurate NUV-NIR photometry (i.e., excluding flagged regions around saturated objects) was obtained for 1558 sources ( $~ 86 \%$ of 1814$), 61$ of which are multiplecomponent radio sources. Hereafter, we refer to this latter sample of 1558 radio sources, which make up $\sim 65 \%$ of the radio sources with $\mathrm{S} / \mathrm{N} \geq 5$ and that were matched to the NUV-NIR catalog, as the "matched radio source sample." For reference, the $1.4 \mathrm{GHz}$ total flux density distributions for the complete radio sample, the matched radio sample, and the subsample with available spectroscopy is shown in the top panel in Figure 3. The distribution of the $i$-band magnitude for the matched radio sample, as well as the spectroscopic subsample, is shown in the bottom panel in Figure 3. It is also worth noting that our cross-correlation is consistent with the results of the maximum likelihood ratio technique applied to VLA-COSMOS sources (P. Ciliegi et al. 2008, in preparation); however, our restrictions for the masked-out regions in the photometric catalog, as well as the optical magnitude limit, are more conservative, as the analysis presented here strongly relies on accurate NUV-NIR photometry.

A further data set that we use in the analysis presented here is the HST ACS point-source information. We extract this information for each radio source in our matched sample by positionally matching the optical counterparts of the radio sources with point-sources identified in the HST ACS F814W source catalog (Leauthaud et al. 2007). Using a matching radius of $0.5^{\prime \prime}$ yields 47 objects in our matched radio sample classified as point sources based on the HST ACS F814W images. The mean distance between the matched objects is only $0.12^{\prime \prime} \pm 0.07^{\prime \prime}$.

\subsection{Radio-Optical Sources with IRAC and MIPS Detections}

We cross-correlate the matched radio source sample with the S-COSMOS-IRAC catalog using a maximum allowed distance to the optical counterparts of our radio sources of $0.5^{\prime \prime}$. (Note that such a cross-correlation allows for a maximum distance between the radio and MIR sources to be $1^{\prime \prime}$.) Such an adopted search radius essentially selects a complete radio-optical-MIR sample with a false match association for the MIR sources of $\lesssim 1 \%$ with the optical counterparts, and $\lesssim 4 \%$ with the radio counterparts. In summary, out of 1558 radio sources in the matched radio sample, $1448(93 \%)$ have secure MIR counterparts.

The $24 \mu \mathrm{m}$ flux densities for all our radio sources were obtained from the COSMOS field observations using the S-COSMOSMIPS shallow survey with a resolution of $6^{\prime \prime}$. Although a relaxed search radius of $5^{\prime \prime}$ was used to find the radio $24 \mu \mathrm{m}$ counterparts, the median distance is only $0.19^{\prime \prime}$ with an interquartile range of $0.16^{\prime \prime}$. About $50 \%$ (799 out of 1558 ) sources in the matched radio sample have a MIPS counterpart at $24 \mu \mathrm{m}$ with a signal-to-noise ratio at or above 3 .

\subsection{Radio-Optical Sources with Pointlike X-Ray Emission}

Using the maximum likelihood ratio technique Brusa et al. (2007) presented the optical identifications of the X-ray point sources
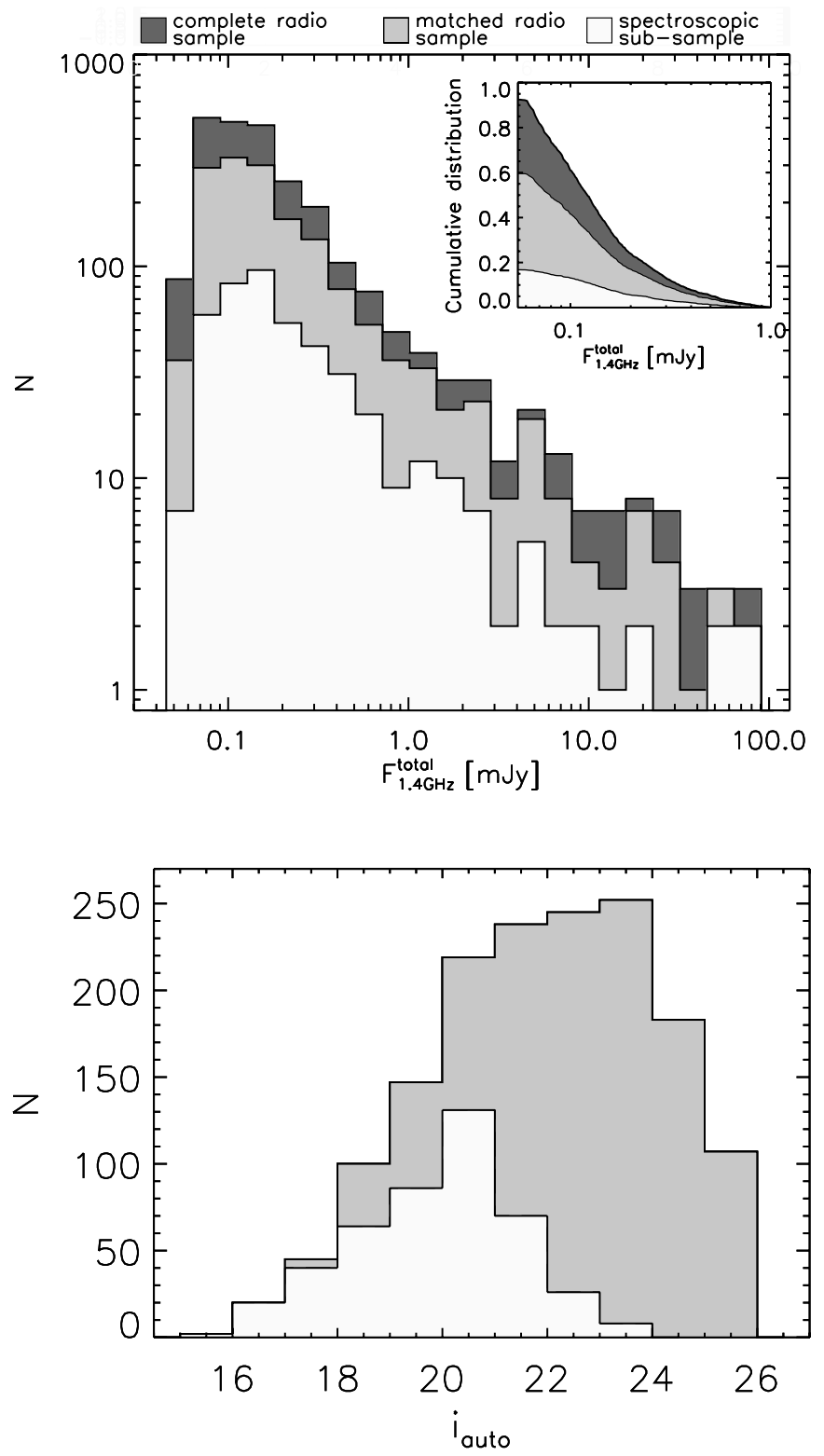

FIG. 3.-Top: Distribution of the total $1.4 \mathrm{GHz}(20 \mathrm{~cm})$ flux density for (1) the complete radio source sample ( $\mathrm{S} / \mathrm{N} \geq 5$; dark gray shaded histogram), (2) the matched radio sample (gray shaded histogram) and (3) the spectroscopic subsample (light gray shaded histogram). The inset shows the cumulative distribution for the three samples computed as a function of decreasing total flux. Note that the spectroscopic subsample fairly represents the faint radio population as a function of the total flux. Bottom: Distribution of the $i$-band magnitude (Subaru where available, otherwise CFHT) for sources in the VLA-COSMOS matched radio source sample, and the spectroscopic subsample in the same notation as in the top panel.

(Cappelluti et al. 2007) detected in the XMM-Newton COSMOS survey (Hasinger et al. 2007). Here we use their identifications to match the sources in our matched radio sample with detected X-ray point sources. Out of 1558 radio sources with optical counterparts, $179(12 \%$ of 1558$)$ are identified as point sources in the X-ray bands. Seventeen of these have multiple counterpart candidates as defined by Brusa et al. (2007). In these cases, if we assume that the radio sources are physically associated with the $\mathrm{X}$-ray sources, then the radio data, which have a significantly better astrometric accuracy, can be used to constrain more precisely the optical counterpart of this given object. A visual inspection of the 17 sources, classified as having ambiguous identifications by M. Brusa et al. (2008, in preparation) strongly suggests that their 
TABLE 1

Multiwavelength Cross-Correlation of Vla-COSMOS 1.4 GHz Radio Sources

\begin{tabular}{|c|c|c|c|c|c|}
\hline Sample Group & Total & $z_{\text {spec }}$ & $\operatorname{IRAC}^{\mathrm{a}}$ & MIPS $^{b}$ & $X M M-N e w t^{\mathrm{c}}$ \\
\hline 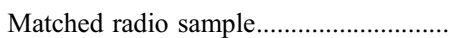 & 1558 & 447 & 1448 & 799 & 179 \\
\hline Stars................... & 2 & 0 & 2 & 2 & 0 \\
\hline QSOs ...................... & 139 & 31 & 122 & 78 & 43 \\
\hline SF galaxies ${ }^{\mathrm{d}}$.. & 340 & 150 & 322 & 280 & 16 \\
\hline AGN galaxies $^{\mathrm{d}} \ldots$ & 601 & 262 & 579 & 267 & 98 \\
\hline 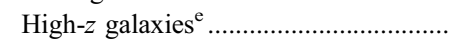 & 476 & 4 & 423 & 172 & 22 \\
\hline Remaining radio sample ......................... & 830 & 73 & 610 & 318 & 31 \\
\hline 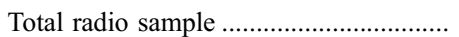 & 2388 & 520 & 2058 & 1117 & 210 \\
\hline
\end{tabular}

Notes.- The matched radio source sample contains sources positionally matched to optical sources with $\mathrm{AB} i \leq 26$ and outside masked-out regions in the NUV-NIR photometric catalog described by Capak et al. (2007). The remaining radio sample has sources (1) without optical counterparts with $i \leq 26(\sim 70 \%)$ or (2) with optical counterparts $(i \leq 26)$ that lie in masked-out areas in the photometric catalog $(\sim 30 \%)$.

${ }^{\mathrm{a}}$ Detected in the Spitzer/IRAC $3.6 \mu \mathrm{m}$ band.

b Detected in the Spitzer/MIPS $24 \mu \mathrm{m}$ band within the shallow MIPS COSMOS survey at or above a signal-tonoise ratio of 3

c X-ray point-sources associated with optical counterparts as described in Brusa et al. (2007).

d Galaxies at redshift $\leq 1.3$.

e Galaxies at redshift $>1.3$.

most probable optical counterparts, reported in the X-ray-optical catalog, are real associations. Hence, we proceed in our analysis taking all $179 \mathrm{X}$-ray-detected point sources to be true counterparts of the objects in the matched radio sample.

\subsection{Radio Sources with Photometrically Flagged} or without Optical Counterparts at Other Wavelengths

In $\S 3.1$ we have defined the matched radio sample which consists of $15581.4 \mathrm{GHz}$ sources that have optical counterparts out to an $i$-band magnitude of 26, and within a radial distance of less than $0.5^{\prime \prime}$. These sources were also required to have the most accurate NUV-NIR photometry, i.e., counterparts within flagged regions due to saturation and blending effects in the NUV-NIR images were excluded. Thus, 830 radio sources remain with no identified optical counterparts within these limits, 256 (i.e., $~ 30 \%$ ) of which have counterparts with $i \leq 26$ that lie in masked-out regions. Hereafter, we refer to this sample of sources as the "remaining radio source sample." We positionally match these sources to the IRAC catalog using a maximum allowed distance of $1^{\prime \prime}$ and find $610(\sim 75 \%)$ matches. Based on Poisson statistics and the source density of the MIR sources, such a search radius essentially includes all true matches with a false contamination rate of $\$ 4 \%$. It is worth noting that more than one-half of the remaining $25 \%$ of the radio objects were independently identified as possible spurious sources, based on visual inspection, while the other half are either located in blended regions in the IRAC images or slightly further away than the allowed 1 " from the position reported in the IRAC catalog (the morphology of the IRAC sources being often extended). Thus, we consider these $\sim 75 \%$ of radio-MIR matches representative of the entire remaining radio population. Out of the 830 sources 318 (i.e., $\sim 40 \%$ ) have MIPS $24 \mu \mathrm{m}$ detections ( $\mathrm{S} / \mathrm{N} \geq 3$ ), and $31(\sim 4 \%)$ have XMM-Newton point-source counterparts (these 31 objects are a subsample of the 256 objects in the flagged regions). In $\S 7$ we analyze the properties of these remaining sources, and their contribution to the population mix in the VLA-COSMOS survey. The summary of the multiwavelength crosscorrelation of the VLA-COSMOS radio sources is given in Table 1.

\section{CLASSIFICATION METHODOLOGY}

Extragalactic radio sources consist of two main populations: $\mathrm{SF}$ and AGN galaxies. We further divide the AGN class into two subclasses: QSOs (often unresolved in optical images, with broad emission lines in their spectra and high optical luminosity) and objects where the AGN does not dominate the entire SED, such as type 2 QSOs, low-luminosity AGNs (Seyfert and LINER galaxies) and absorption-line AGNs (resolved in optical images, with both broad, narrow, or no emission lines in their optical spectra). Throughout the paper, we mostly refer to the latter subclass only as "AGNs."

The commonly adopted and well-calibrated tool for disentangling SF galaxies from low-luminosity AGNs (Seyfert and LINERs) is the optical spectroscopic diagnostic diagram (Baldwin et al. 1981), which is based on two emission-line flux ratios $\left(\left[\mathrm{O}_{\text {III }} 25007\right] / \mathrm{H} \beta\right.$ vs. [N II 26584$] / \mathrm{H} \alpha$; hereafter the BPT diagram; see also Veilleux \& Osterbrock 1987; Rola et al. 1997; Kewley et al. 2001). This diagnostic tool has been extensively used in the past for a successful separation of local SF and AGN galaxies (Sadler et al. 1999; Kauffmann et al. 2003; Brinchmann et al. 2004; Obrić et al. 2006; Smolčić et al. 2006). However, as spectroscopic observations are very expensive in terms of telescope time, especially when large numbers of faint objects need to be observed, alternative methods for the separation of SF from AGN galaxies, which eliminate the need for spectroscopy, must be invoked. Here we develop such a method (hereafter the rest-frame color-based classification method), which we apply in the following sections to identify SF and AGN galaxies in the VLA-COSMOS matched radio sample. The main idea of our method is drawn from the findings that the overall NUV-NIR SED of galaxies is a one-parameter family and that spectral diagnostic parameters, such as line strengths, appear to be well correlated with the overall galaxy's SED (see Obrić et al. 2006; Smolčić et al. 2006). In particular, Smolčić et al. (2006) have found a tight correlation between rest-frame colors of emissionline galaxies and their position in the BPT diagram. This correlation thus provides a powerful tool for disentangling SF from AGN galaxies using only photometric data, i.e., rest-frame colors, and we use it as the key of our rest-frame color-based classification method.

\subsection{Calibration of the Rest-Frame Color-based Classification Method in the Local Universe}

\subsubsection{The Local Spectroscopic Sample}

In order to obtain insight into the efficiency of the classification method, based on the rest-frame color ( $P 1$; see below), we 
construct a large sample of well-known low-redshift galaxies $(0.01<z<0.3)$, whose properties are assumed to present well the properties of the galaxies in the VLA-COSMOS matched radio sample. The local sample was generated from the SDSS main galaxy sample, positionally matched to sources detected in the $1.4 \mathrm{GHz}$ NVSS survey. In addition, a subsample of galaxies detected with IRAS was constructed (see Obrić et al. 2006 for details about the cross-correlation of the SDSS, NVSS, and IRAS catalogs). The SDSS/NVSS sample contains 6966 galaxies and the SDSS/NVSS/IRAS sample of 875 galaxies with available SDSS optical spectroscopy. The computation of rest-frame colors $(P 1, P 2)$ for these galaxies is presented in Smolčić et al. (2006). The rest-frame colors $P 1$ and $P 2$ optimally quantify the distribution of galaxies in the rest-frame color-color space. They are derived from the modified Strömgren photometric system $(u z, v z, b z, y z)$ encompassing the wavelength range of $3200-$ $5800 \AA$ Å; (see also Smolčić et al. 2006; Odell et al. 2002) via a two-dimensional principle component analysis in color-color space. A more detailed description of the colors, including their equational form, is given in Appendix A.

It is noteworthy to mention that given (1) the detection limits and (2) the areal coverage of the NVSS and VLA-COSMOS surveys, both surveys observe approximately the same populations of objects, although over different redshift intervals (see Fig. 1 in Schinnerer et al. 2007). Assuming that evolutionary effects with redshift do not significantly alter the reliability of the identification method presented below, at least out to $z=1.3$, this makes the local sample of galaxies representative of the galaxies in the VLA-COSMOS matched radio sample.

Based on spectral-line properties we separate the local sample into three classes of objects: AGN, SF galaxies, and composite objects, where the latter are considered to have a comparable contribution of both star formation and AGN activity. First, galaxies with emission lines in their spectra are separated into these three classes using their position in the BPT diagram (see bottom panel in Fig. 4). ${ }^{19}$ Second, as the galaxies that have no emission lines in their spectra cannot be star forming (see also $\S 4.1 .3$ for a discussion of this point), and as all of the objects in the SDSS/NVSS sample are observed to have $1.4 \mathrm{GHz}$ emission which arises either from an AGN or star formation activity in a galaxy, we define galaxies without emission lines in their spectra as AGNs (see also Best et al. [2005], who classified these types of objects as absorption-line AGNs).

\subsubsection{Completeness and Contamination due to the Photometric Selection}

In the top panel in Figure 4 we show the distribution of $\sim 3400$ SDSS/NVSS emission-line galaxies in the $(P 1, P 2)$ restframe color-color diagram. The color code is determined by the position of a galaxy in this plane. The bottom panel shows the BPT diagram for the same galaxies with the colors adopted from the top panel. For these radio luminous galaxies, a strong correlation exists between their rest-frame optical colors and emissionline properties, in particular between $P 1$ and $\log ([\mathrm{N}$ II 26584$] / \mathrm{H} \alpha)$. We want to stress that the SDSS/NVSS galaxies with no emission lines in their spectra have typically red $P 1$ colors, with a median $P 1$ value of 0.46 and an interquartile range of 0.13 (see also, e.g., Fig. 12 in Smolčić et al. 2006). This implies that the restframe color $P 1$ can be used as an efficient separator between AGNs

19 Note that the classification of AGN, SF, and composite galaxies are based on the BPT diagram changed compared to the one used in Smolčić et al. (2006).
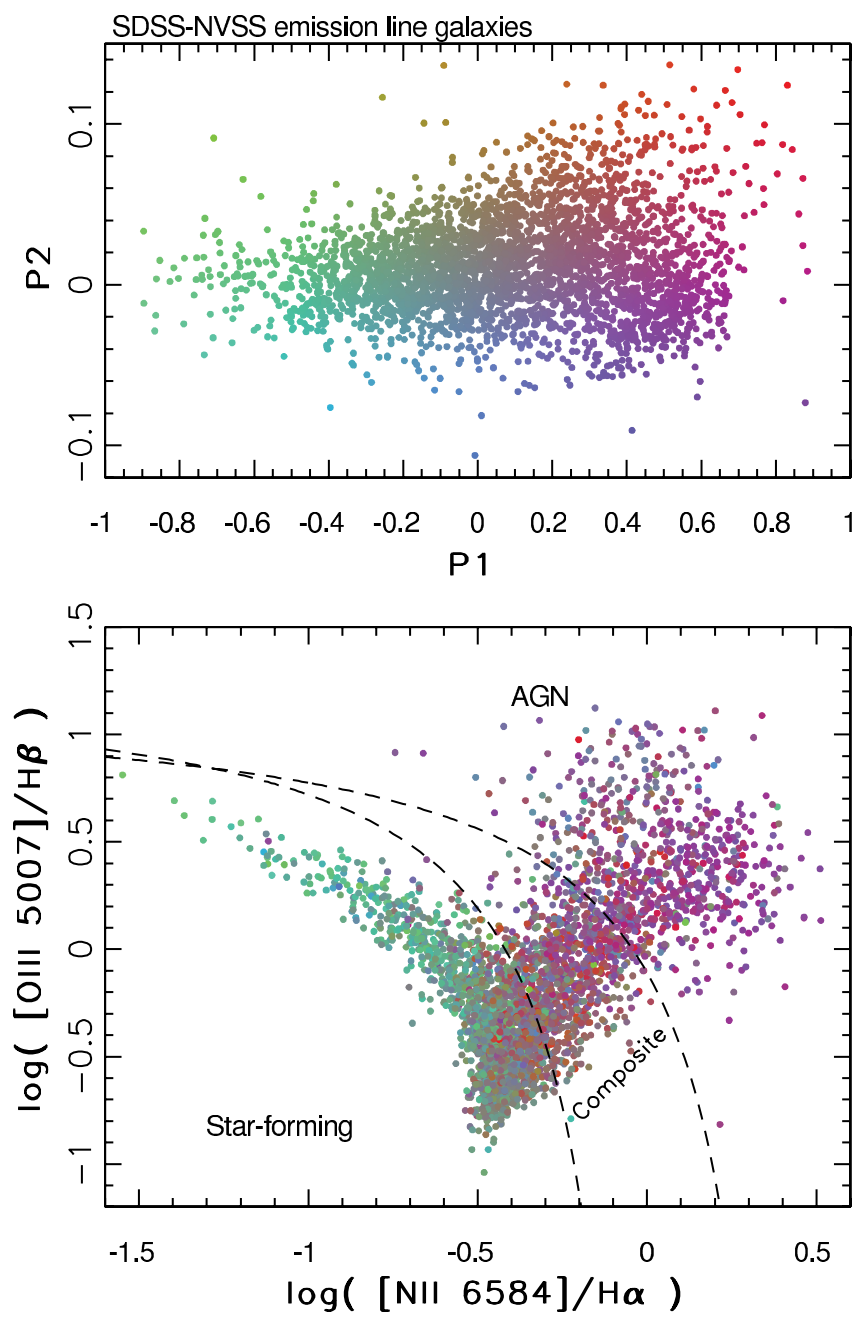

FIG. 4.-Top: Distribution of $\sim 3,400$ SDSS emission-line galaxies, in the redshift range of 0.01 to 0.3 , drawn from the DR1 "main" spectroscopic sample, which were also detected by the $1.4 \mathrm{GHz}$ NVSS survey, in a diagram constructed with the principal rest-frame colors $(P 1, P 2)$. Each dot corresponds to one galaxy, and the color code is determined by the position in this plane. Bottom: Distribution of these galaxies in the (BPT) diagram (Baldwin et al. 1981). The bottom dashed line separates the regions populated by SF and composite galaxies (Kauffmann et al. 2003). The top dashed line separates the regions populated by composite and AGN galaxies (Kewley et al. 2001). The dots are colored according to their $P 1$ and $P 2$ values given in the top panel. Note the strong correlation between the restframe colors and the emission-line flux ratios (see text for details).

and SF galaxies in samples where these two types of galaxies are the two dominant populations.

Smolčić et al. (2006) argued that the $P 2$ color is a proxy for the galaxies' dust content, with higher values of $P 2$ corresponding to higher dust attenuation. However, as the dynamic range of $P 2$ is very narrow, we would rather base our selection on the $P 1$ color alone. Hereafter, based on a compromise between completeness and contamination of the photometrically selected samples of galaxies, we choose a color-cut of $P 1=P 1$ cut as the dividing photometric value between SF galaxies $(P 1 \leq 0.15)$ and AGNs $(P 1>0.15)$. Given this boundary we infer that the sample of photometrically selected SF galaxies is $85 \%$ complete and contaminated by AGNs and composite objects at the $20 \%$ and $10 \%$ levels, respectively. Vice versa, the photometrically selected sample of AGNs is $90 \%$ complete and contaminated by SF and composite galaxies at the $5 \%$ and $15 \%$ level, respectively (see Appendix B1 for computational details). We use these estimates 
in the following analysis to statistically correct the photometrically selected SF and AGN samples in the VLA-COSMOS survey.

\subsubsection{Is the Rest-Frame Color-based Selection Method Biased against Dusty Starburst Galaxies?}

One of the main advantages of radio observations is that the intrinsic physical properties that drive the radio emission can be derived without any need for dust-extinction corrections (as radio emission passes freely through dust). In particular, radio observations provide a dust-unbiased view of star formation (see Condon 1992 for a review; see also Haarsma et al. 2000). Hence, it is important to address whether our rest-frame color-selection technique misses out dusty starburst galaxies. In order to do this, we study a subsample of SDSS/NVSS galaxies that were also detected with IRAS at IR wavelengths.

First we address the composition of the "missed" $\sim 15 \%$ of the SF galaxies (note that the photometrically selected SF galaxy sample is $85 \%$ complete) in order to show that our selection does not introduce biases against the most luminous starburst galaxies. About $30 \%$ of the SF galaxies with $P 1>0.15$ have IRAS detections, which is consistent with the fraction of IRAS detections in the SF galaxy sample with $P 1 \leq 0.15$. This suggests that the composition of the missed SF galaxies is not significantly different from the composition of the selected SF galaxies. Second, the fractions of luminous and ultraluminous IR galaxies (LIRGs and ULIRGs, respectively) in the spectroscopically classified SF galaxy samples with $P 1 \leq 0.15$ and $P 1>0.15$ appear to be consistent with each other.

And third, the completeness and contamination of the SDSS/ NVSS/IRAS galaxy subsamples selected using the rest-frame color-based classification method is fairly consistent with the properties of the entire SDSS/NVSS sample (see Appendix B2 for details). All of this implies that the selection criteria for SF and AGN galaxies adopted on the basis of the analysis of the full SDSS/NVSS sample works almost equally efficiently for the IRAS-detected subsample. Furthermore, only $\sim 10 \%$ and $\sim 5 \%$ of SF LIRGs and ULIRGs galaxies, respectively, are omitted by the method (see Appendix B2 for details).

It is noteworthy that in the entire SDSS/NVSS/IRAS sample only 48 objects (i.e., $\sim 5 \%$ ) were identified as absorption-line AGNs, i.e., having no emission lines in their optical spectra. It is possible that very high dust obscuration may suppress the detection of emission lines in the optical spectrum. A visual search for signatures of $\mathrm{H}$ II regions in the 48 SDSS color-composite images suggested that at most $30 \%$ of these galaxies may possibly be undergoing star formation (e.g., possible galaxy merger or extended morphology). Therefore, only a negligible fraction of less than $1.5 \%$ of the SDSS/NVSS/IRAS galaxies may be so heavily dust-obscured that no emission lines would be detected in their optical spectra.

In order to test this issue further, we have synthesized the $P 1$ color for the "standard" dusty starburst galaxies M82 (a typical LIRG), and Arp 220 (the prototypical ULIRG) using spectral templates given by Polletta et al. (2007). The optical to NIR part of these templates was generated using the stellar population synthesis code GRASIL (Silva et al. 1998). The derived $P 1$ colors for M82 and Arp 220 are 0.078 and 0.149 , respectively, implying that M82 would not have been missed by the rest-frame color-based classification method, while Arp 220, although close to the adopted limit in $P 1$, still lies within our selection criterion (note that in reality observational photometric errors of M82- and Arp 220like objects will introduce a scatter in $P 1$; see also $\S 4.2 .2$ ). Based on the tests presented above we conclude that our rest-frame color- based classification method is not significantly biased against dusty starburst galaxies.

\subsection{Application of the Rest-Frame Color-based Classification Method to the Multiwavelength Photometry of the VLA-COSMOS Radio-Optical Galaxies}

In the previous section we have studied and calibrated the rest-frame color-based classification method using the local galaxy sample. Assuming that evolutionary effects with redshift do not significantly affect the reliability of the classification, we can safely apply it to the galaxies in the VLA-COSMOS $1.4 \mathrm{GHz}$ matched radio sample ( $\S 5.4$ ) to separate SF from AGN galaxies at intermediate redshifts. However, first we need to derive the restframe $P 1$ color from the observed SED of the VLA-COSMOS galaxies. We do this via high-resolution SED fitting, described in $\S 4.2 .1$, and we test the accuracy of the rest-frame color synthesis in $\S 4.2 .2$.

\subsubsection{Derivation of Rest-Frame Colors}

In order to estimate the rest-frame color $P 1$ for each galaxy in the matched radio sample that we do not classify as a star or QSO (see $\S 5$ ), we use the GOSSIP (Galaxy Observed Simulated SED Interactive Program) software package (Franzetti 2005), designed for fitting a galaxy's SED to a set of chosen spectral models. The SED of the galaxies in our sample, which we use for fitting, extends from $3500 \AA$ to $2.5 \mu \mathrm{m}$ (comprising six photometric passbands), and we fit to each observed SED a realization of $\sim 100,000$ spectra built using the Bruzual \& Charlot (2003, hereafter $\mathrm{BC} 03$ ) stellar synthesis evolutionary models.

Star formation histories have been parameterized by an underlying continuous star formation history (decaying exponentially), and randomly superimposed bursts (see also Kauffmann et al. 2003; Kong et al. 2004; Salim et al. 2007). We cover ages between $100 \mathrm{Myr}$ and $13.5 \mathrm{Gyr}$, specific star formation rates (star formation rate per unit galaxy stellar mass) between $10^{-15}$ and $3.93 \times 10^{-8} \mathrm{yr}^{-1}$ and metallicities from one-tenth to twice solar.

For each object in our sample the model spectra in our library are redshifted to the galaxy's measured redshift (spectroscopic where available, otherwise photometric), then each spectrum is convolved with the observed filter response function, ${ }^{20}$ and then fitted to the available observed photometric data, using a direct $\chi^{2}$-minimization procedure. Output parameters, such as, e.g., restframe colors, stellar mass or the $4000 \AA$ break, are taken from the best-fit model spectrum. In order to derive physically meaningful output parameters, we restrict the fitting procedure to models that have an age smaller than that of the universe at the galaxy's redshift.

\subsubsection{Accuracy of the Derived Rest-Frame Colors}

The synthesized, relative to observed, colors and magnitudes for the VLA-COSMOS galaxies, computed as described above, are reproduced with a satisfying accuracy of $\sim 0.07$ and $\sim 0.04$, respectively. To further test the accuracy of the derived rest-frame color, we synthesize $P 1$ for a sample of $\sim 1700$ local SDSS/NVSS galaxies. For these galaxies the $P 1$ colors, computed from their spectrum (with an accuracy of better than $0.03 \mathrm{mag}$; see Smolčić et al. 2006), are also available. Thus, comparing $P 1$ synthesized via SED fitting with the reference $P 1$ derived from the spectrum gives us a direct measure of the achieved accuracy, shown in Figure 5 (top). The rms scatter is $\sim 0.1$.

\footnotetext{
20 The COSMOS filter response curves can be found online at http://www .astro.caltech.edu/ capak/cosmos/filters.
} 

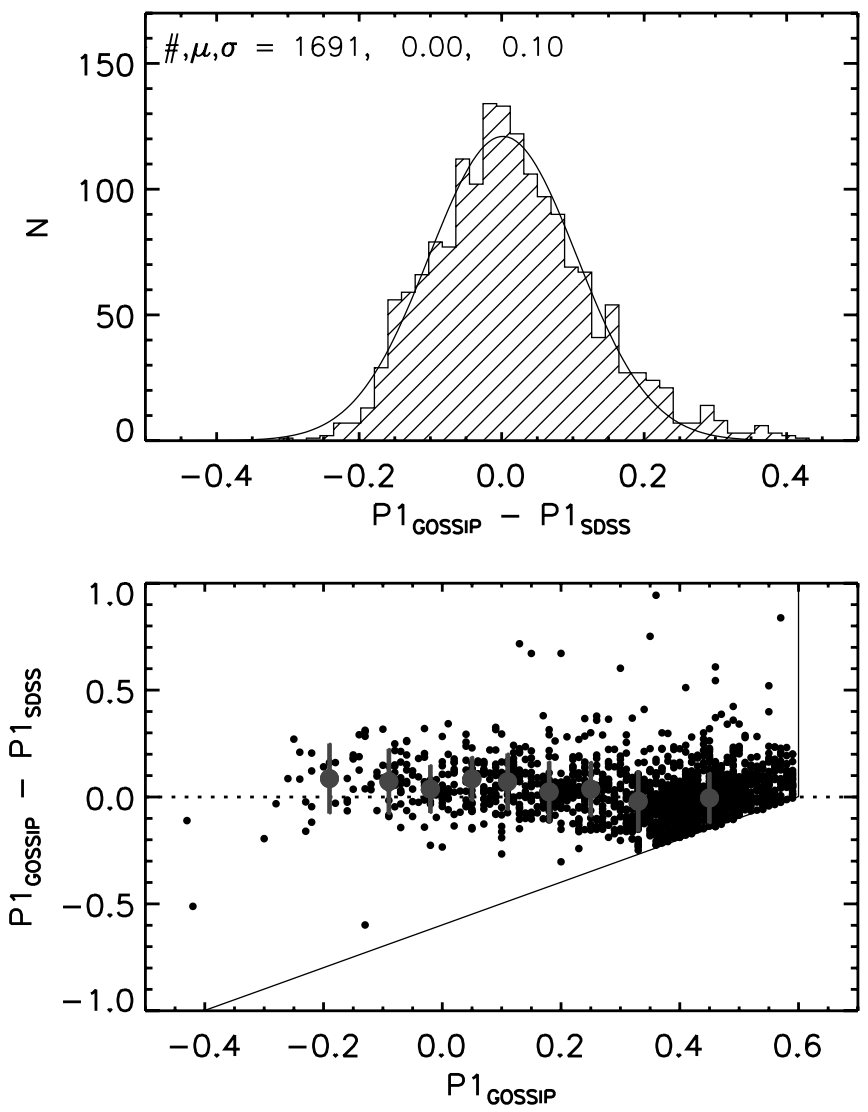

FIG. 5.-Comparison of the $P 1$ color synthesized from the SDSS spectrum $\left(P 1_{\mathrm{SDSS}}\right.$ that has a typical error of 0.03$)$ and via SED fitting $\left(P 1_{\mathrm{GOSSIP}}\right)$ for $\sim 1700$ SDSS/NVSS galaxies from the DR1 "main" spectroscopic sample (top). The $P 1$ color is reproduced with a spread of $\sim 0.1$. The bottom panel shows the difference in the $P 1$ color as a function of $P 1_{\mathrm{GOSSIP}}$. The thin solid lines show the upper $P 1$ limits of 0.6 , imposed for this analysis. Note the slight systematic trend in the derived $P 1$ color as a function of $P 1_{\mathrm{GOSSIP}}$ (the large circles are the median offsets, with indicated $1 \sigma$ error bars). The trend presumably arises due to the presence of strong emission lines in the rest-frame wavelength range of $3800-5800 \AA$, that are not taken into account in the $\mathrm{BC} 03$ models. We use these offsets to correct for these systematics in the derivation of $P 1$ for the clean radio sample (see text for details). [See the electronic edition of the Supplement for a color version of this figure.]

In the bottom panel in Figure 5 we show the difference of the $P 1$ color as a function of the synthesized (GOSSIP) $P 1$ color for the SDSS/NVSS galaxies. A slight systematic trend is present as a function of the derived $P 1$ color. This trend presumably arises from the presence of strong emission lines in the galaxies' SEDs (in the rest-frame wavelength range that is used to derive $P 1$ ), which are not taken into account in the $\mathrm{BC} 03$ model spectra. In our further analysis of the VLA-COSMOS data, we use the median offset, shown in the bottom panel in Figure 5, to correct the derived $P 1$ color, and we consider the synthetic $P 1$ color to be accurate to $\sim 0.1 \mathrm{mag}$. However, an error of $0.1 \mathrm{mag}$ for $68 \%$ of the galaxies, and 0.2 for $95 \%$, may substantially alter the SF/AGN selection, introducing the largest uncertainty for the galaxies that have $P 1$ colors close to the chosen $P 1$ boundary. To account for these uncertainties, we simulate the $P 1$ errors using a randomly drawn Gaussian distribution with a standard deviation of 0.1 centered at zero (Fig. 5, top). These errors are then added to the galaxies' $P 1$ colors derived from the best-fit template in the SED fitting, and the SF/AGN selection (see $\S 5.4$ ) is applied. By repeating this procedure 10,000 times we obtain a robust statistical distribution of the number of selected SF and AGN galaxies yielding $356 \mathrm{SF}$ and 585 AGN galaxies (mean) with a rms scatter of only 7.
Applying our SF/AGN selection using the $P 1$ distribution obtained from the best-fit template in the SED fitting yields $340 \mathrm{SF}$ and $601 \mathrm{AGN}$ galaxies (see $\S 5.4$ ). Thus, 5\% less SF galaxies are selected. This is easily understood as the blue tail of the $P 1$ distribution contains a smaller number of galaxies than there are in the prominent red peak (see, e.g., Fig. 24). Therefore, a normal error distribution will systematically scatter more galaxies to the blue $P 1$ region, than to the red one. We conclude that the photometric errors of the synthesized $P 1$ color introduce a number uncertainty of $\sim 5 \%$ in favor of SF galaxies. Although $\sim 5 \%$ is not significant, it is necessary to keep this bias in mind in the analysis of the population mix of submillijansky radio sources $(\S 7.2)$.

\section{CLASSIFICATION OF THE VLA-COSMOS SOURCES IN THE MATCHED RADIO SAMPLE}

In this section we present the classification of the entire VLACOSMOS matched radio sample into star candidates (§ 5.2), QSOs (§ 5.3), SF, AGN, and high-z galaxies (§ 5.4). We begin with a summary of the sample definitions ( $(5.1)$.

\subsection{Outline and Nomenclature}

In $\S 4.1$ (see also Appendix B) we present the calibration and effectiveness of the rest-frame color-based classification method for separating galaxies dominated by star formation from those dominated by AGN activity. For this we have used the SDSS "main" spectroscopic sample, a pure galaxy sample that by definition does not contain any starlike objects (Strauss et al. 2002). This, obviously, implies that the same effectiveness of the method can only be reached if the rest-frame color-based classification method is applied to a comparable sample, i.e., galaxies only. However, our VLA-COSMOS matched radio sample consists not only of galaxies, but also of stellar-like sources, where the latter are either stars or quasi stellar objects. Therefore, we classify the sources in the matched radio sample into five subtypes: (1) star candidates, (2) quasi stellar objects (QSOs), (3) active galactic nuclei (AGNs), (4) star-forming (SF), and (5) high-redshift ( $z>1.3$; high- $z$ ) galaxies. The latter three subtypes compose our "VLA-COSMOS galaxy sample." The properties of each subtype are summarized as follows.

Stars.- Point sources in the optical, with their SEDs best fit using a stellar template.

OSQs.-Point sources in the optical (stellar-like SEDs are excluded; see above). This criterion essentially requires that the emission of the nucleus in the optical strongly dominates over the emission of the host galaxy. Thus, this sample predominantly contains broad-line AGNs (i.e., type 1 AGNs), with power-law spectra in the optical.

$A G N s$.-Galaxies (not point sources) whose rest-frame color properties are consistent with properties of AGNs $(P 1>0.15$, $\mathrm{X}$-ray luminosity above $10^{42} \mathrm{ergs} \mathrm{s}^{-1}$ if X-ray detected). This selection requires that the optical emission either shows signs of both, the emission from the central AGN as well as the emission from the underlying host galaxy, or only the latter. Thus, this sample essentially includes Seyfert and LINER types of galaxies, as well as absorption-line AGNs, and we limit it to redshifts of $z<1.3$.

$S F$ galaxies.-Galaxies whose rest-frame color properties are consistent with properties of SF galaxies $(P 1 \leq 0.15)$. Thus, the emission of these galaxies is dominated by the emission originating from regions of substantial star formation. This sample is also limited to redshifts $\leq 1.3$.

High-z galaxies.-Galaxies (not point-sources) with redshifts beyond $z=1.3$. 

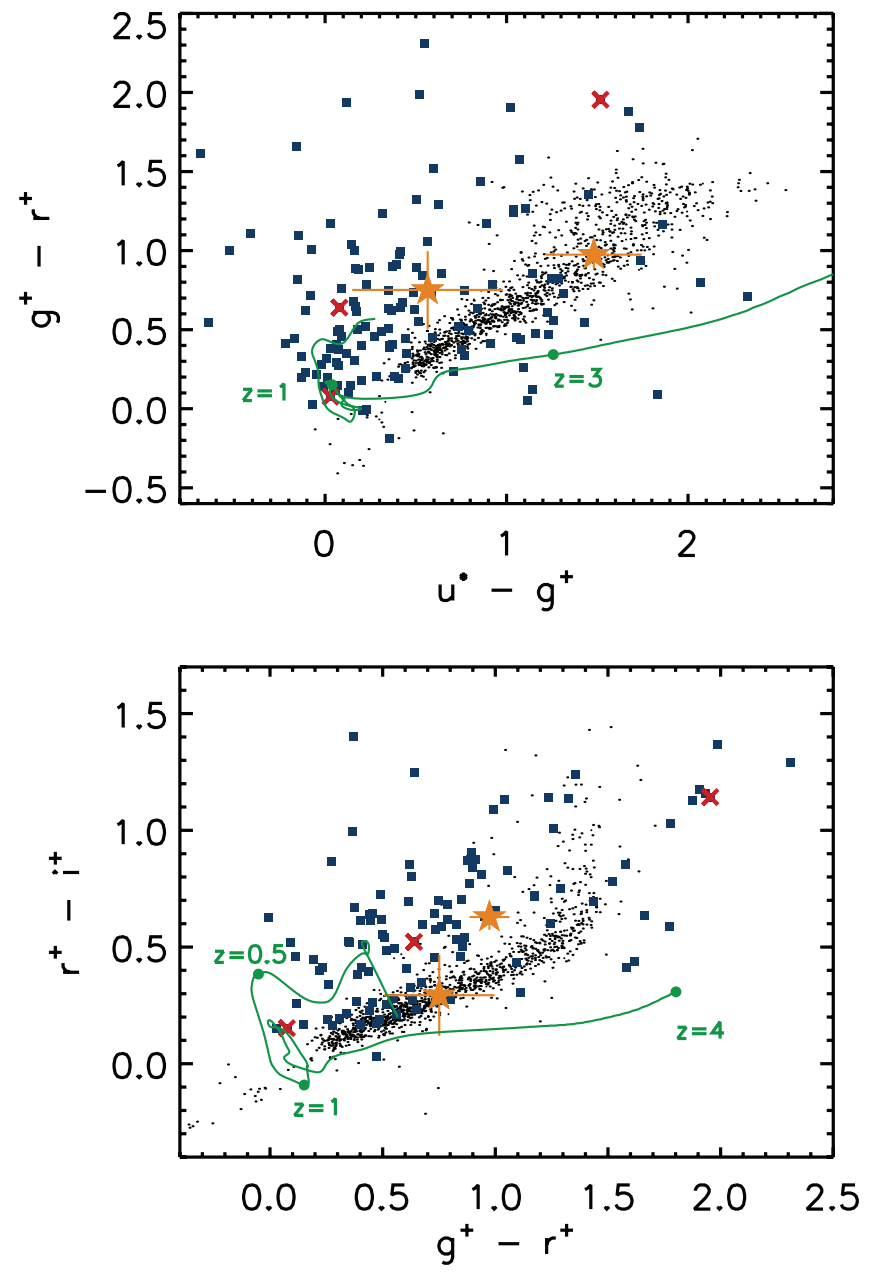

FIG. 6.-Color-color diagrams for COSMOS stars and VLA-COSMOS QSOs (see text for details). The stars (black dots) form a narrow, well defined, locus in each diagram. The yellow star symbols show two objects detected in the VLACOSMOS radio survey, identified as star candidates (see text for details). VLACOSMOS objects classified as QSOs by our selection criteria (blue squares) are also shown, and they occupy the "standard" QSO regions in these diagrams (see text for details). The red crosses show objects classified as a QSO by our technique, yet their spectra were identified as red galaxies. QSO evolutionary tracks are shown to guide the eye (green curved lines).

\subsection{Star Candidates}

In order to identify star candidates in the VLA-COSMOS matched radio source sample, we make use of the COSMOS stellar cata$\log$ (L. Tasca et al. 2008, in preparation), that was constructed from the HST ACS catalog (Leauthaud et al. 2007) using stellar templates to fit the entire SED of each source. In Figure 6 we show the color-color distribution for $\sim 2000$ objects in the COSMOS field securely classified as stars (with photometric errors better than 0.05 ), which form well-defined loci in the broadband colorcolor diagrams. Cross-correlating our matched radio sample with the COSMOS stellar catalog yields only two objects detected in the radio regime that are consistent with having stellar properties. The color properties of these objects are shown in Figure 6. Within the error bars they are consistent with the main stellar loci. Note, however, the $r^{+}-i^{+}$color excess of one of the star candidates in the $r^{+}-i^{+}$vs. $g^{+}-r^{+}$color-color diagram (Fig. 6, middle), which suggests consistency with properties of, e.g., cataclysmic variables (e.g., Szkody et al. 2002, 2003), or unresolved binary star systems containing a white dwarf and a late-type star (e.g., Smolčić et al. 2004). The best-fit stellar templates for these

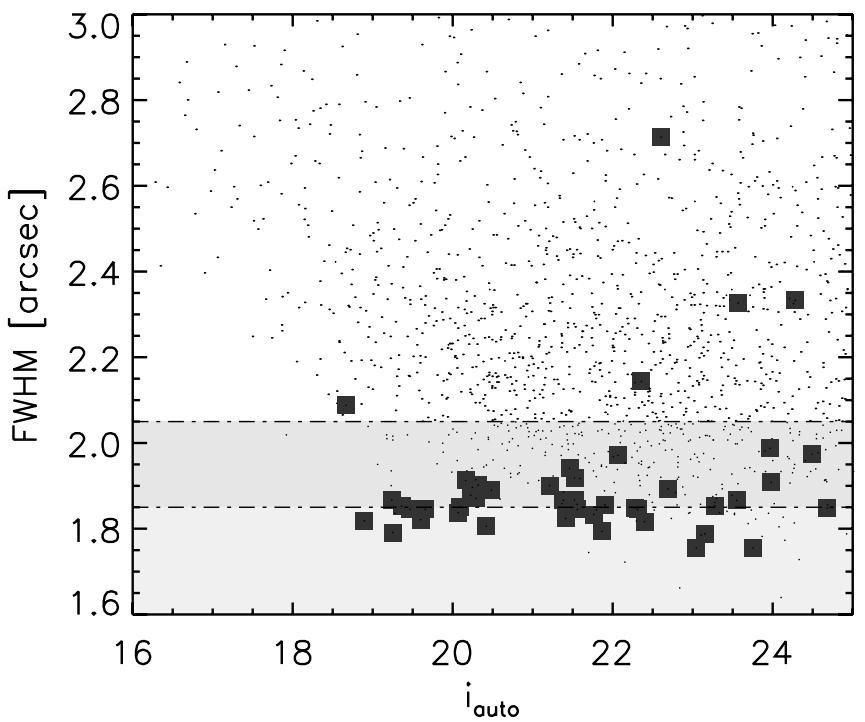

FIG. 7.-FWHM of the optical counterparts of the matched radio sources (dots) as a function of $i$ band magnitude, taken from the COSMOS photometric catalog (Capak et al. 2007). Gray squares represent the HST ACS point sources in the sample. Unresolved sources form a separated locus in this diagram. We identify QSOs by requiring that a source is optically compact by choosing an absolute separation value of FWHM $\lesssim 1.85^{\prime \prime}$ (below bottom dash-dotted line; light gray shaded area). In the range of FWHM $\in\left(1.85^{\prime \prime}, 2.05^{\prime \prime}\right]$ (between dash-dotted lines; dark gray shaded area), we identify objects as QSOs only if they were visually confirmed to be point-source dominated (see text for details).

objects were taken from the PHOENIX library (Hauschildt et al. 1997) and represent dwarfs with effective temperatures in the range of 4100 to $5000 \mathrm{~K}$ and $\log (g)$ in the range of 3-3.5. The $1.4 \mathrm{GHz}$ total flux densities for these two objects are 126 and $152 \mu \mathrm{Jy}$, and the corresponding $i$-band $\mathrm{AB}$ magnitudes are 25.34 and 23.28, respectively. It is noteworthy that both objects have IRAC counterparts, but no associated X-ray emission. We consider these two sources to be star candidates; however, a more detailed analysis (using for example spectroscopy), which is beyond the scope of this paper, would be needed to verify this. Such a low fraction of identified stars is consistent with star detection rates in other deep radio surveys (e.g., Fomalont et al. 2006). The two star candidates in our radio sample form only $\sim 0.1 \%$ of the VLA-COSMOS radio sources, and we exclude them from our sample for further analysis.

\subsection{Quasi Stellar Objects}

\subsubsection{Identification Based on Morphology}

In order to identify QSOs in our matched radio sample we rely on an optical classification, rather than using X-ray emission, due to the much higher sensitivity of the observations in the optical (5 $\sigma$ sensitivity limit in the Subaru $i$-band is 26.2; see Capak et al. 2007). For example, if one would select AGNs relying purely on, e.g., X-ray-to-optical flux ratios, which are generally greater than 0.1 for both broad- and narrow-line AGNs (e.g., Maccacaro et al. 1988; Alexander et al. 2001), with our optical limit of $i=26$ (corresponding to $r^{+}$of $\sim 27$ ) the depth of the X-ray point-source detection would have to be about 2 orders of magnitude deeper than it currently is in order to select a complete sample of AGNs. Furthermore, a clear distinction between broad- and narrow-line AGNs would not be possible. Hence, here we identify a QSO by requiring that a given source in the matched radio sample is optically compact. In Figure 7 we show the fitted $i$-band FWHM of the sources in the COSMOS photometric catalog (Capak et al. 2007) as a function of their $i$-band magnitude. Point sources 

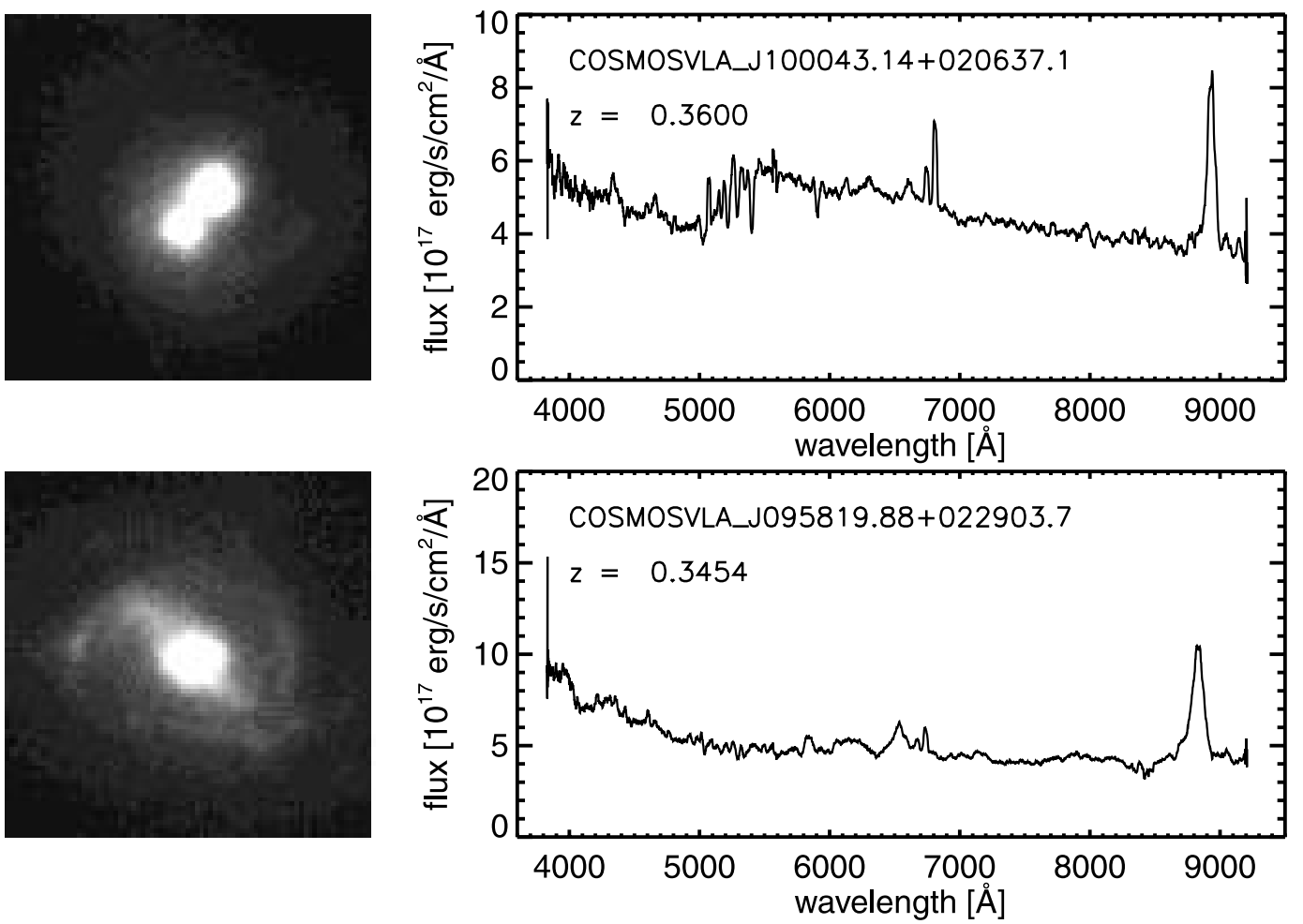

FIG. 8.-HSTACS stamps ( $4^{\prime \prime}$ on a side; left) and the corresponding SDSS spectra (smoothed using $20 \AA$ wide bins; right) for two sources that were spectroscopically

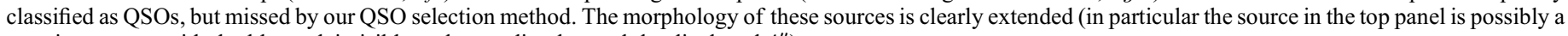
merging system with double nuclei visible and extending beyond the displayed $4^{\prime \prime}$ ).

(Fig. 7, black squares), selected from the HST ACS catalog (Leauthaud et al. 2007), form a locus in this plane, separated from the area occupied by extended sources. However, the pointsource locus is fairly scattered, especially at faint magnitudes and, thus, makes a single automatic cut at a certain FWHM value inefficient. For this reason, we classify sources within the FWHM range of $1.85^{\prime \prime}-2.05^{\prime \prime}$ as QSOs only if their optical HST morphology was visually confirmed to be point-source dominated. However, we consider all sources below FWHM of $1.85^{\prime \prime}$ to be QSOs. We further supplement this sample with 12 objects that were classified as point sources in the HST ACS catalog, but do not satisfy the above criteria.

In summary, out of 1558 objects, we identify 139 (i.e., $11.5 \%$ ) as QSOs. In Figure 6 we show their broadband $\left(u^{*}, g^{+}, r^{+}, i^{+}\right)$ color-color properties. As expected, the nonstellar emission of the selected objects confines them to regions typical for QSOs, which are separated from the main stellar loci in these diagrams (e.g., Brusa et al. 2007; Richards et al. 2002). A minor fraction of these objects lie on the stellar loci. However, in the $B z K$ diagnostic diagram, which is an efficient tracer for stars (Daddi et al. 2004), these sources are offset from the stellar locus, verifying their nonstellar nature.

As AGN-dominated systems usually have soft X-ray-to-optical flux ratios in the range of about 0.1-10 (e.g., Maccacaro et al. 1988; Alexander et al. 2001), we can use the X-ray-to-optical flux properties of the identified QSOs to further test our selection criteria. In our QSO sample 43 objects were detected as X-ray point sources, and their X-ray-to-optical flux ratios are consistent with the expected values. The median $r^{+}$magnitude for these sources is 21.3. For the remaining QSO candidates, which were not detected in the X-ray, the median $r^{+}$is 24.4. Therefore, these sources are also consistent with the expected X-ray-to-optical flux ratios; however, beyond our X-ray point-source detection limit $\left(10^{-15} \mathrm{ergs} \mathrm{cm}^{-2} \mathrm{~s}^{-1}\right.$ in the soft band $)$. We conclude that the independent analysis of the X-ray properties of our selected QSOs verifies the validity of our selection.

\subsubsection{Spectroscopic Verification}

A subsample of 31 objects of the 139 previously identified QSOs have available optical spectroscopy with secure classifications ( Trump et al. 2007; Prescott et al. 2006; Colless et al. 2001; Schneider et al. 2005), and only three of these objects were classified as red galaxies ( Trump et al. 2007), while all the others have AGN classifications. The three galaxies classified as ellipticals were identified as QSO candidates by our method based on visual/ morphologic classification, which suggests the presence of dominating nuclear emission. The color properties of two of theses objects (Fig. 6, red crosses in the top panel) are also consistent with the color properties of quasars. ${ }^{21}$ Thus, we conclude that the selected QSO sample is not significantly ( $\lesssim 10 \%)$ affected by contamination of non-QSO objects.

In order to assess the completeness of the selected QSO sample, we search for objects that are spectroscopically classified as QSOs and "missed" by our classification method. Our criteria yielded 139 objects classified as QSOs in the matched radio sample, and in the remainder of the sample (i.e., the 1417 sources that were not classified as star candidates or QSOs) spectroscopic classifications are available for 397 objects. Out of these, nine were spectroscopically classified as QSOs. Two SDSS examples, for which COSMOS HST ACS imaging is available, are shown in Figure 8 . They obviously show extended optical emission,

\footnotetext{
${ }^{21}$ For example, in the $u^{*}-g^{+}$vs. $g^{+}-r^{+}$color-color diagram (Fig. 6, top)
} red galaxies would occupy the top right quadrant (see, e.g., Strateva et al. 2001). 
and a substantial light component arises from the host galaxy itself. The median redshift of these nine objects is only 0.4. It is noteworthy that all of these objects have X-ray point-source detections, and all except one have X-ray luminosities higher than $10^{42} \mathrm{ergs} \mathrm{s}^{-1}$. Therefore, these galaxies will be selected into our AGN class, hence not contaminating the SF galaxy sample (see $\S 5.4$ ). As the spectroscopic subsample fairly represents the full matched radio sample (see Fig. 3), we conclude, based on the above analysis, that the sample of identified QSOs is about $80 \%$ complete. As expected, the incompleteness is mostly due to relatively low-redshift, low-luminosity AGNs.

\subsection{Star-forming and AGN Galaxies}

In the previous sections we have identified two star candidates and 139 QSOs in the matched radio sample. We refer to the 1417 remaining sources in the matched radio source sample as the "galaxy sample." Before applying the rest-frame color-based classification method to our VLA-COSMOS galaxies in order to separate SF from AGN galaxies, we restrict the galaxy sample to 941 galaxies with redshifts $\leq 1.3$, as (1) the photometric redshifts are less reliable beyond this redshift, and (2) the library of $\mathrm{BC} 03$ model spectra that we use for the SED fitting may not be appropriate for fits beyond this redshift, as the distribution of priors was set to optimally match this intermediate-redshift range. Hereafter, we call the sample of 476 galaxies with redshifts greater than 1.3 high-redshift (high-z) galaxies.

We perform an SED fit using GOSSIP (as described in $\S 4.2$ ) for each of the 941 objects in the matched radio "galaxy" sample out to $z=1.3$. The distribution of the rest-frame color $P 1$ for these galaxies is shown in Figure 9. The distribution is very similar to that of the local sample (Fig. 24, top) with a peak at $P 1 \sim 0.4$ (AGNs) and a prominent tail toward bluer values (SF galaxies). We inspected the behavior of the median value of the synthesized $P 1$ color for the entire $z \leq 1.3$ galaxy sample as a function of redshift, and we found no significant evolution in the median color. We reached the same conclusion analyzing the median $P 1$ colors of the SF and AGN subpopulations. This invariance in rest-frame color is consistent with the results presented in Barger et al. (2007), who found very little rest-frame color variation with redshift of the radio-optical sample in the Hubble Deep Field-North. This implies that a fixed cut in the rest-frame color can safely be applied to the entire galaxy population out to $z=1.3$.

The SED fitting was performed via a $\chi^{2}$-minimization procedure. The median value of the reduced $\chi^{2}$ of the SED fits, computed using the best-fit model spectrum, is 0.6 with an interquartile range of 2 . Only $10 \%$ of the fitted objects have reduced $\chi^{2}$ values above 5 , and only $5 \%$ have values above 10 . A visual inspection suggests that these galaxies are either nearby galaxies, which are resolved and often saturated in the Subaru $i$ band, or QSO contaminants. While the latter have predominantly blue $P 1$ colors, the synthesized $P 1$ color for the first class of galaxies still appears to be a valid tracer for the SF/AGN separation, and therefore, we do not reject them from the sample. In order to select SF and AGN galaxies we require that the synthesized $P 1$ color is $\leq 0.15$ and $>0.15$, respectively. However, to improve our selection at this point we make use of the X-ray properties in the soft band of the 114 galaxies that were detected as X-ray point sources. Namely, if the soft X-ray luminosity of an object is greater than $10^{42} \mathrm{ergs} \mathrm{s}^{-1}$, we consider it to be an AGN, regardless of its $P 1$ color. Note that this criterion is expected to reduce the contamination of the SF sample by objects with blue rest-frame colors, such as QSOs missed by our selection. Out of the $114 \mathrm{X}$-ray-detected sources, 77 have $\mathrm{X}$-ray luminosities greater than the above-given value, and 37 out of these 77 have a synthetic $P 1$ color $\leq 0.15$. In summary, our

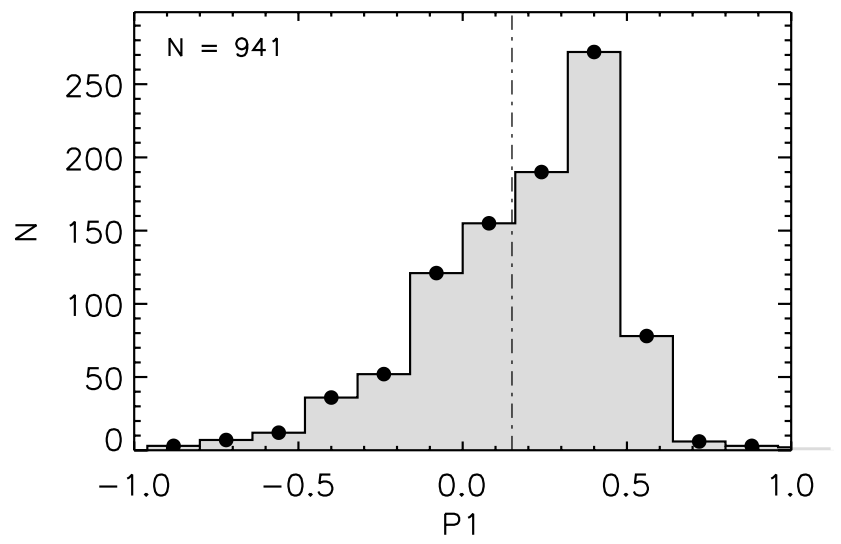

FIG. 9.-Distribution of the synthesized rest-frame $P 1$ color, corrected for the systematics (Fig. 5, bottom), for the objects in the matched radio galaxy sample with $z<1.3$. Note that the expected distribution from our local sample, showing a prominent tail toward bluer values of $P 1$, is reproduced. The dashdotted line indicates our chosen boundary for separating SF from AGN galaxies.

selection yields $340 \mathrm{SF}$ and $601 \mathrm{AGN}$ galaxies in our matched radio galaxy sample with $z \leq 1.3$. We analyze these galaxies further in $\S 7$.

\section{COMPARISON WITH OTHER SELECTION METHODS}

In this section we compare our classification method, which we have applied to an intermediate-redshift population, with other classification methods used for both local and intermediateredshift populations in the literature (Lacy et al. 2004; Stern et al. 2005; Best et al. 2005). We also study the $24 \mu \mathrm{m}$ properties of our radio sources and their correlation to the $1.4 \mathrm{GHz}$ emission.

\subsection{6-8 $\mu \mathrm{m}$ Color-Color Diagnostics}

QSOs, whose UV-NIR continuum is dominated by a power law, tend to be redder than other types of galaxies in the MIR. Hence, they occupy a distinct region in the MIR color space, and several color-color criteria were suggested for their selection (Lacy et al. 2004; Stern et al. 2005). In Figure 10 we compare our classification method with those proposed in the MIR using a subsample of the matched radio sources that were also detected with IRAC ( $\sim 90 \%$ have IRAC counterparts; see $\S 3.2)$. We indicate the QSO (Fig. 10, dots), AGN (thin contours) and SF (thick contours) galaxies selected using our method. The dashed lines in the top and bottom panels in Figure 10 show the color-color criteria proposed by Lacy et al. (2004) and Stern et al. (2005), respectively, for the selection of broad-line AGNs. As expected, the majority of objects selected as QSOs by our method falls within this region, reassuring the efficiency of the classification method presented here. There are several QSO candidates outside these regions, which is not surprising as the suggested "quasar regions" do not select a $100 \%$ complete sample of QSOs, and a certain amount of outliers is expected (see Stern et al. [2005] for a discussion of this point). In $\S 5.3$ we have inferred that our selected sample of QSOs is not significantly contaminated by different types of objects, which is affirmed by this independent analysis.

Stern et al. (2005) showed that at redshifts of $\lesssim 1$ galaxies span a large range in the $m_{5.8}-m_{8.0}$ color, which is consistent with the horizontal extent of our selected SF and AGN galaxies (see Fig. 10, bottom). However, typical low-luminosity AGNs and starburst galaxies cannot be clearly divided using these diagnostic diagrams (see also Fig. 19, color evolutionary tracks). Nonetheless, elliptical galaxies (which correspond to our class of absorption-line 

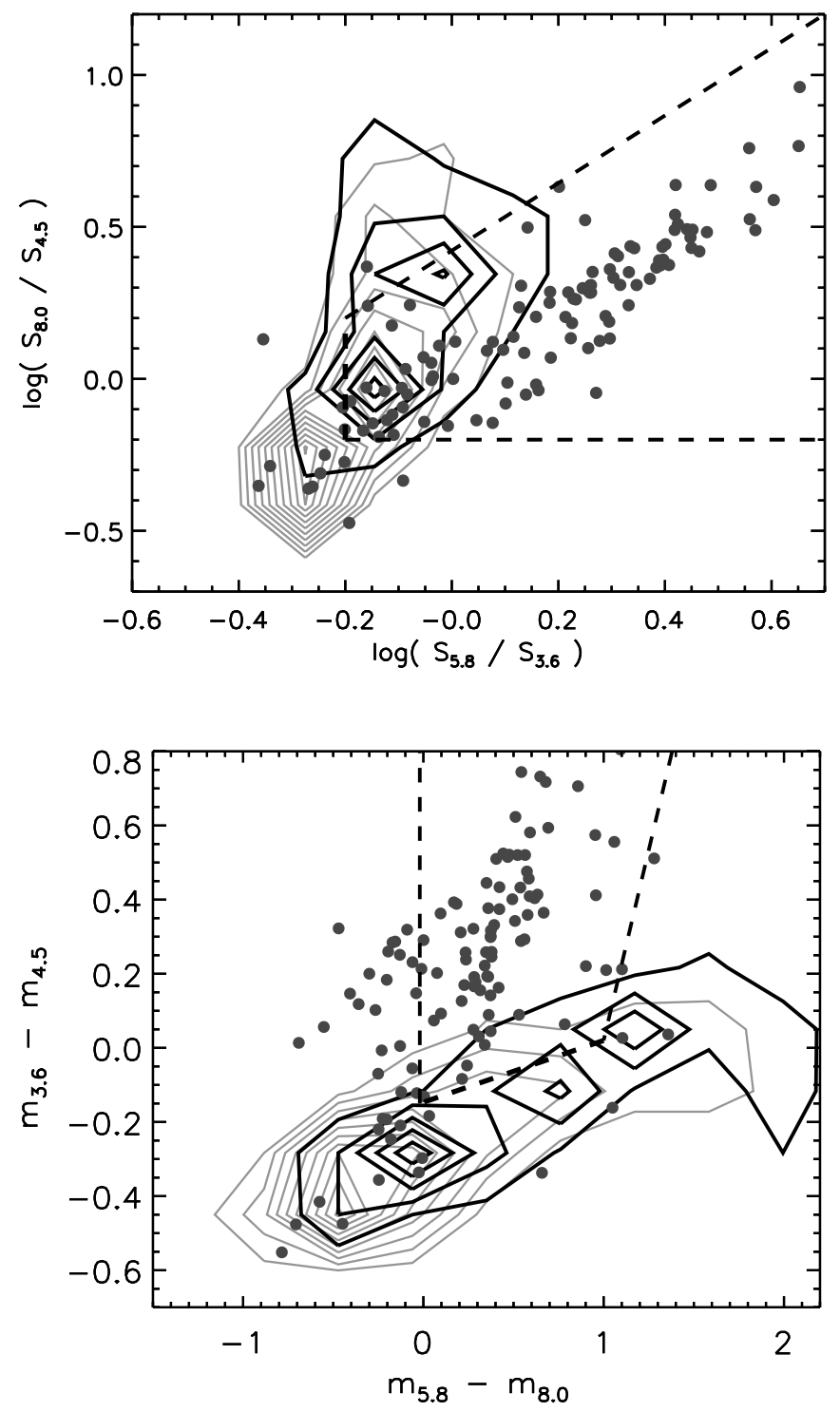

FIG. 10.- IRAC color-color diagrams in two representations (top and bottom panel) used as diagnostic tools for the separation of QSOs (broad-line AGNs) from "normal" galaxies (Lacy et al. 2004; Stern et al. 2005). Filled circles display the 122 QSOs selected by our morphologic method that have IRAC detections; thin and thick contours show the 579 AGN and 322 SF galaxies separated by our rest-frame color-based classification method, respectively, that have IRAC counterparts. The contour levels in both panels are in steps of 7 starting at 7 . The dashed lines in the top and bottom panels show the empirically selected regions for identifying broad-line AGNs proposed by Lacy et al. (2004) in the top panel and Stern et al. (2005) in the bottom panel, respectively.

AGNs) tend to occupy the bottom left regions in both diagrams, and close to these regions the distributions of our identified AGNs peak. On the other hand, the peak of the distribution of our selected SF galaxies in these diagrams is clearly displaced from that for AGNs. We would like to stress that this independently confirms that indeed two different populations of galaxies have been selected. The differences in the MIR properties of our SF and AGN galaxies affirm the strength of the rest-frame colorbased classification method.

Furthermore, Stern et al. (2005) showed that narrow-line AGNs appear spread out in both the QSO and galaxy regions, which is also a result of our selection method (note that the selected AGNs are present in both regions). The last point we want to stress is that the SF galaxy locus in these diagrams is also consistent with the expected colors, as a "contamination" by SF galaxies of the QSO

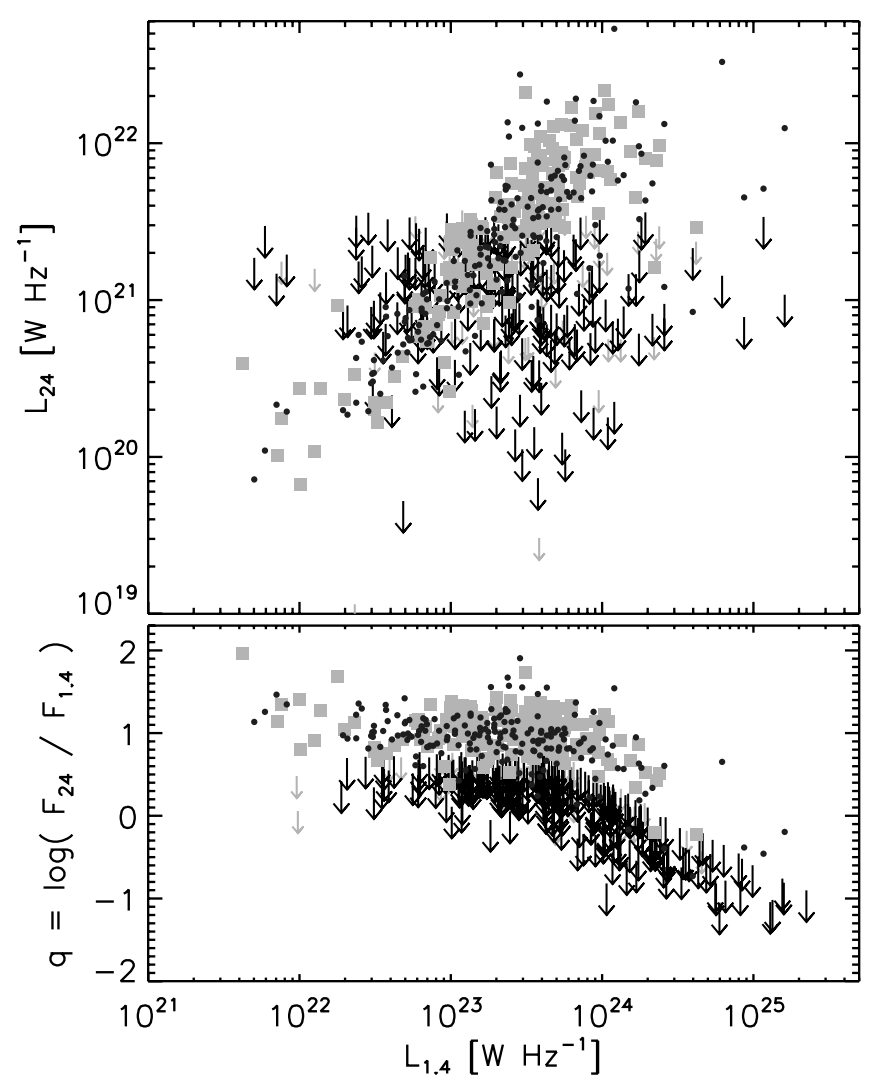

FIG. 11.-Top: $24 \mu \mathrm{m}$ luminosity vs. $1.4 \mathrm{GHz}$ luminosity for our selected SF ( gray squares) and AGN (black dots) galaxies. Upper $24 \mu \mathrm{m}$ luminosity limits for the radio SF (18\%) and AGN (56\%) galaxies that were not detected at $24 \mu \mathrm{m}$ are indicated by gray and black arrows, respectively. Bottom: The $q$-parameter, defined as the logarithm of the $24 \mu \mathrm{m}$ to $20 \mathrm{~cm}$ flux ratio, as a function of $20 \mathrm{~cm}$ luminosity for SF (gray squares) and AGN (black dots) galaxies. Upper limits are indicated by arrows, as in the top panel. Note the much larger scatter in $q$ for AGN than for SF galaxies.

locus is expected, especially close to the boundary. In summary, the classification method presented here agrees remarkably well with the expected properties of QSOs, AGNs, and SF galaxies at intermediate redshifts in the MIR range encompassing 3.6-8 $\mu \mathrm{m}$.

\section{2. $24 \mu \mathrm{m}$ Properties: The $24 \mu \mathrm{m}$ Radio Correlation}

A tight MIR (as well as far- and total-IR) radio correlation is expected for SF galaxies, while "radio-loud" AGNs are expected to strongly deviate from it (e.g., Condon 1992; Bell 2003; Appleton et al. 2004). The $60 \mu \mathrm{m}$ radio correlation for low-luminosity AGNs was studied by Obrić et al. (2006) in the local universe. Based on a selection using the BPT diagram they have shown that also lowluminosity AGNs follow a tight FIR-radio correlation, however, with a slightly different slope and a larger scatter than SF galaxies. In this section we investigate the $24 \mu \mathrm{m}$ radio correlation for our selected SF and AGN galaxies. In particular, if our SF/AGN separation method is successful, then a difference in the $24 \mu \mathrm{m}$ compared to $20 \mathrm{~cm}$ properties is expected to be seen for the two populations.

Our rest-frame color-based classification method has identified 340 SF galaxies. Out of these $82 \%$ (280) were detected at $24 \mu \mathrm{m}$ with a signal-to-noise ratio $\geq 3$. On the other hand, out of 601 selected AGNs, only 44\% (267) have a MIPS $24 \mu \mathrm{m}$ detection with $\mathrm{S} / \mathrm{N} \geq 3$. In Figure 11 we show the $24 \mu \mathrm{m}$ versus 1.4 GHz luminosity (Fig. 11, top) for our SF and AGN galaxies, where the $24 \mu \mathrm{m}$ data were not $k$-corrected. A correlation between the two luminosities exists for both types of objects detected at 


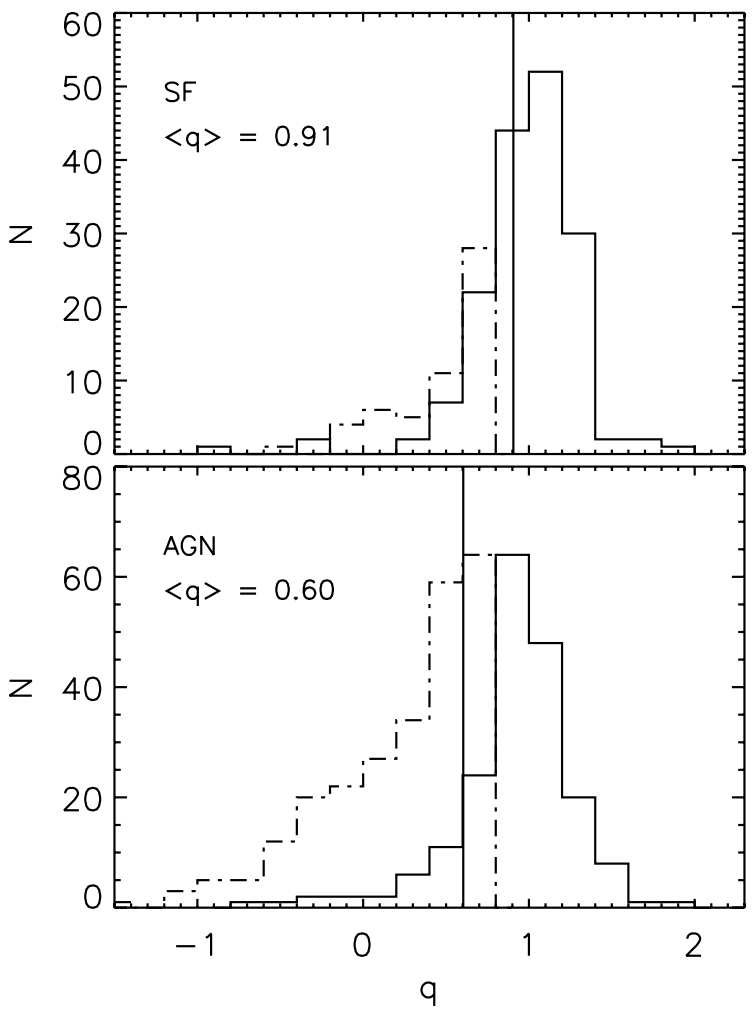

FIG. 12.-Distribution of the $q$-parameter (see also Fig. 11) for SF (top) and AGN (bottom) galaxies. In both panels the solid-line histograms show the distribution of the $1.4 \mathrm{GHz}$ sources detected at $24 \mu \mathrm{m}$ while the dash-dotted-line histograms represent the distribution of the upper limits of $q$ obtained for the radio sources that were not detected at $24 \mu \mathrm{m}$ (see text for details). The solid vertical line in each panel designates the median value of the entire distribution, also listed in the top left in each panel.

$24 \mu \mathrm{m}$, although on average for a given $L_{1.4 \mathrm{GHz}}$ the $24 \mu \mathrm{m}$ luminosity is slightly lower for AGN than for SF galaxies (see also below). For the SF and AGN galaxies that were not detected at $24 \mu \mathrm{m}$, we have computed upper limits of the $24 \mu \mathrm{m}$ luminosity using the detection limit of the S-COSMOS MIPS shallow survey which is $0.3 \mathrm{mJy}$. These limiting luminosities are also shown in Figure 11. Note that for AGN galaxies, as 56\% of them are not detected at $24 \mu \mathrm{m}$, the scatter in the correlation is significantly increased by these objects.

To quantify the correlation, we derive the classical $q$-parameter (e.g., Condon 1992) as the logarithm of the $24 \mu \mathrm{m}$ to $1.4 \mathrm{GHz}$ observed flux ratios. This parameter essentially measures the slope of the correlation, and in the bottom panel in Figure 11 we show it as a function of $L_{1.4 \mathrm{GHz}}$ for our SF and AGN galaxies, with indicated upper limits (derived as described above). The $q$-parameter seems to show a decreasing trend with increasing radio luminosity. However, this trend is dominated by the objects that have only estimated upper limits and, therefore, may be mimicked by the flux limits of the samples. A more detailed analysis of this issue is beyond the scope of this paper.

The distribution of the $q$-parameter for SF and AGN galaxies is shown in Figure 12, for galaxies detected at $24 \mu \mathrm{m}$ and those which only have upper limits. The median $q$-value for SF galaxies is $0.91 \pm 0.05$ with a scatter of $\sim 0.3$ when all objects (also the upper limits) are taken into account. On the other hand, the median $q$-value for the AGN galaxies is $0.60 \pm 0.02$, significantly lower than for SF galaxies. We also find a larger spread in $q(\sim 0.4)$ for the AGN population. Note, however, that the spread quoted here should be considered somehow tentative, especially for AGNs,

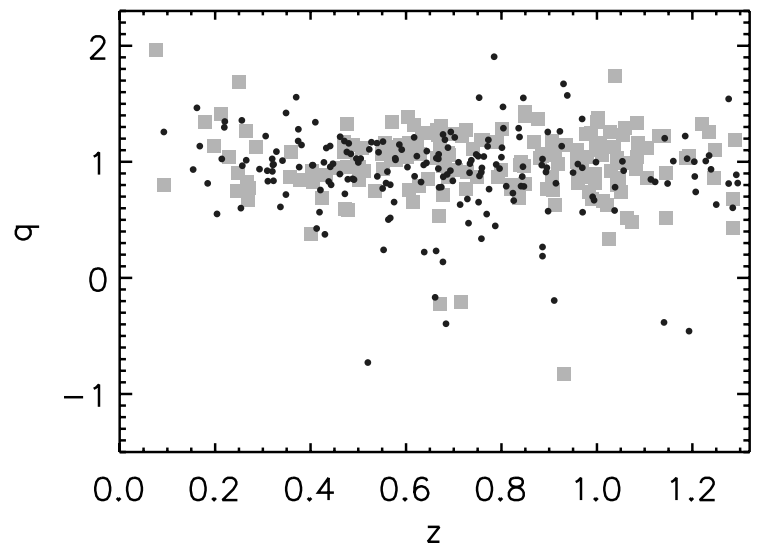

FIG. 13.- Parameter $q$ as a function of redshift for SF (gray squares) and AGN (black dots) galaxies. Note that $q$ does not depend on redshift, implying that the $24 \mu$ m radio correlation holds out to high redshift (see text for details).

as the exact $q$-values for the fraction of objects not detected at $24 \mu \mathrm{m}$ are not known. Nonetheless, this does not affect the estimates of the median values. Our $q$-parameter derived for SF galaxies is remarkably consistent with the one inferred by Appleton et al. (2004) at $24 \mu \mathrm{m}$. Combining Spitzer MIPS and VLA observations in the First Look Survey with optical spectroscopy, they have found a $q$-value of 0.84 with a spread of 0.28 (with no $k$-corrections applied). They have also shown that the FIR luminosities of AGN tend to be lower for a given radio luminosity, consistent with our findings here.

Finally, in Figure 13 we show the $q$-parameter as a function of redshift for our SF and AGN galaxies; $q$ does not depend on redshift, both for SF and AGN galaxies, implying that the MIR radio correlation with the same slope is valid out to high redshifts $(z \sim 1.3)$. This result is again consistent with those presented in Appleton et al. (2004). In summary, the above results have shown that the $24 \mu \mathrm{m}$ radio correlation has different properties for our selected SF and AGN galaxies, which verifies the efficiency of our rest-frame color-based classification method.

\subsection{Selection Based on Optical Spectroscopic Properties, Radio Luminosity, and Stellar Mass}

Best et al. (2005) defined a sample of $\sim 3000$ local $(0.01<$ $z<0.3)$ galaxies from the SDSS DR2 "main" spectroscopic sample matched with sources above $5 \mathrm{mJy}$ detected in the NVSS survey. They further divided the radio-optical source sample into AGN and SF galaxies, making use of the galaxies' location in the plane spanned by the $4000 \AA$ break $\left[D_{n}(4000)\right]$ and radio luminosity $\left[L_{1.4 \mathrm{GHz}}\right]$ normalized by stellar mass $\left[M_{*}\right]$. In Figure 14 we compare our rest-frame color-based classification method with the one used by Best et al. (2005) in the local universe. The $D_{n}(4000)$ versus $\log \left(L_{1.4 \mathrm{GHz}} / M_{*}\right)$ distribution for all galaxies in the matched radio source sample is shown in Figure 14. The $1.4 \mathrm{GHz}$ luminosity for these galaxies was derived, and $D_{n}(4000)$ and $M_{*}$ from the best-fit template from the SED fitting (see $\S 4.2 .1$ ). The average errors are indicated. The dashed line corresponds to the separation between SF and AGN galaxies proposed by Best et al. (2005) and the two types of symbols designate the SF (Fig. 14, squares) and AGN (dots) galaxies identified by our rest-frame color-based classification method. We want to note that Best et al. calibrated their separation method using a slightly different selection of objects in the BPT diagram (see their Fig. 9) with respect to the one we use here. Therefore, a perfect correspondence between our method and that of Best et al. is not to be expected, even if our derived quantities were absolutely accurate. The area 


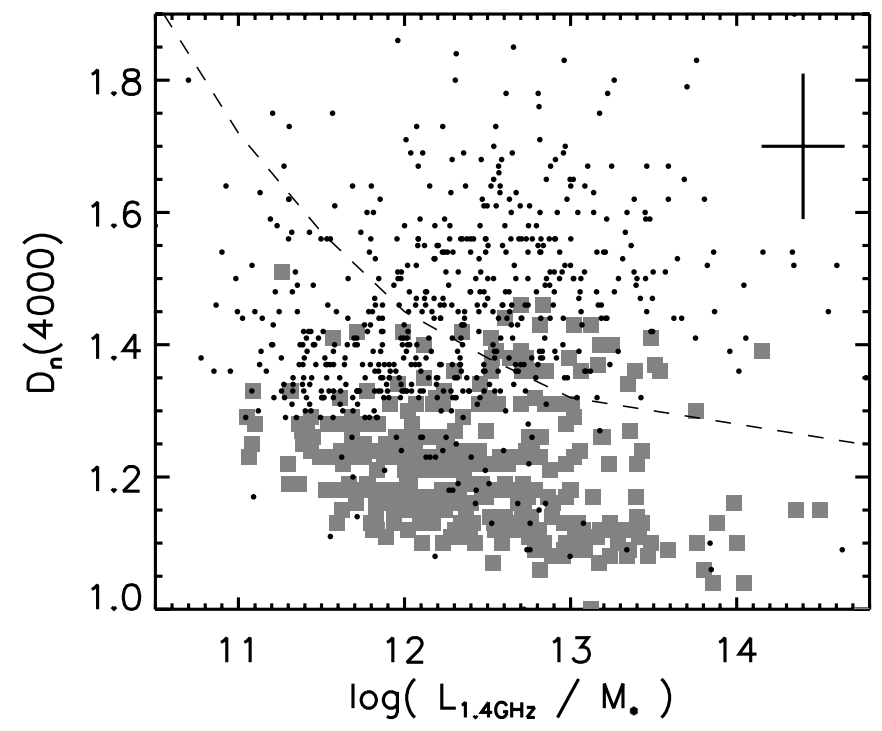

FIG. 14. - The $4000 \AA$ A break, $D_{n}(4000)$, vs. $1.4 \mathrm{GHz}$ radio luminosity normalized by stellar mass, $L_{1.4 \mathrm{GHz} / M}$ for galaxies in the matched radio sample. $M^{*}$ and $D_{n}(4000)$ were synthesized via SED fitting by GOSSIP. The black and gray symbols show objects classified by our rest-frame color-based classification method as AGN and SF galaxies, respectively. The dashed line corresponds to the separation proposed by Best et al. (2005) for local galaxies. The average errors of the synthesized quantities are shown in the top right. A good consistency between the two selection methods exists, given the large error bars, as well as a slightly different selection rational used here and in Best et al. (2005; see text for details).

in the $D_{n}(4000)$ versus $\log \left(L_{1.4 \mathrm{GHz}} / M_{*}\right)$ plane where the major disagreement is expected, due to the different selections in the BPT diagram, is in the range of $1.4<D_{n}(4000)<1.6$, and $11<$ $\log \left(L_{1.4 \mathrm{GHz}} / M_{*}\right)<12$. This is the region where a larger fraction of Seyfert and LINER galaxies is located (see Fig. 9 in Best et al. 2005), and, different from Best et al., we define these galaxies exclusively as AGNs. In this region in Figure 14, we indeed see the largest disagreement between the two classifications. Furthermore, the existence of objects with $D_{n}(4000)<1.3$ that we classify as AGNs is not surprising, but it rather reflects the dual properties of composite objects, which in this case were classified as AGNs by our rest-frame color-based classification method. We also want to note that the average error in the synthesized $D_{n}(4000)$ [derived from comparison with the spectroscopic and synthetic $D_{n}(4000)$ in the local sample] is fairly large and, thus, prevents a more detailed comparison between the two selection methods. Overall, given the error bars and the difference in the basic selections of the two methods, as well as the fact that our $D_{n}(4000)$ values are not spectral measurements on the data, but values taken from the best-fit template, we conclude that our rest-frame color-based classification method agrees well with that proposed by Best et al. (2005) in the local universe. In summary, our rest-frame colorbased classification method for separating SF from AGN galaxies agrees well with other selection schemes, proposed in the literature, which are based both on MIR colors and optical spectroscopic diagnostics.

\section{DISCUSSION: THE COMPOSITION OF THE FAINT RADIO POPULATION}

In previous sections we have presented, tested, and discussed in detail the photometric classification method which we used to separate the matched radio source sample into stars, QSOs, and $\mathrm{SF}, \mathrm{AGN}$, and high- $z$ galaxies. In this section we discuss the properties of the "population mix" in the VLA-COSMOS survey. In
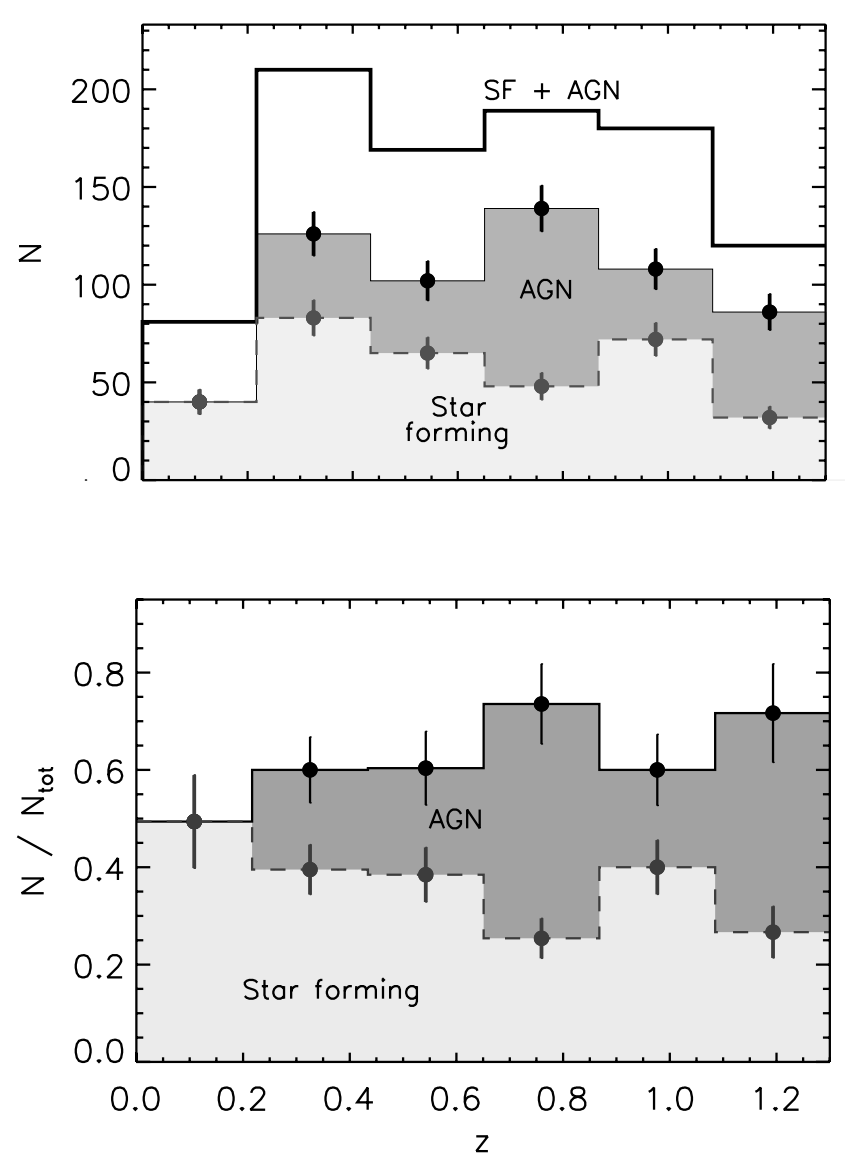

FIG. 15.- Top: Redshift distribution of (1) the galaxies in the matched radio sample out to $z=1.3$ (thick line), (2) the 340 selected SF galaxies (light gray shaded histogram), and (3) 601 identified AGNs (dark gray shaded histogram). Bottom: Fractional distribution of the SF and AGN galaxies compared to the total number of galaxies in the matched radio sample out to $z=1.3$ as a function of redshift. In both panels Poisson errors are indicated.

$\S 7.1$ we describe the redshift and luminosity distributions of the selected SF and AGN galaxies, and in $\S 7.2$ we study the contribution of different source types to the submillijansky radio population. We show, based on the matched radio sample, as well as on the remaining radio sources with no optical counterparts (brighter than $i=26$ ), that SF galaxies do not dominate the submillijansky sources, but that the majority of these sources is rather composed of AGNs and QSOs.

\subsection{The Redshifts and Luminosity Distributions of $S F$ and AGN Galaxies Out to $z=1.3$}

The rest-frame color-based classification method yielded $340 \mathrm{SF}$ and 601 AGN galaxies out to redshifts of 1.3. In the top panel of Figure 15 we show the redshift distribution for these galaxies using redshift bins 0.217 in width. We use such wide redshift bins to assess the average properties of the radio population, reducing the effects of fluctuations due to the strong and narrow overdensities which are known to exist in the COSMOS field (Scoville et al. 2007a; Finoguenov et al. 2007; Smolčić et al. 2007b). Poisson errors are indicated for each bin. The deficit of galaxies at the low-redshift end reflects the relatively small comoving volume sampled by the $2 \mathrm{deg}^{2}$ area of the COSMOS field at these redshifts. The decline in the number of sources at the high-redshift end, on the other hand, reflects the detection limit of the VLACOSMOS survey. The redshift distribution of the number of SF galaxies seems to be more uniform than the one for AGNs, 


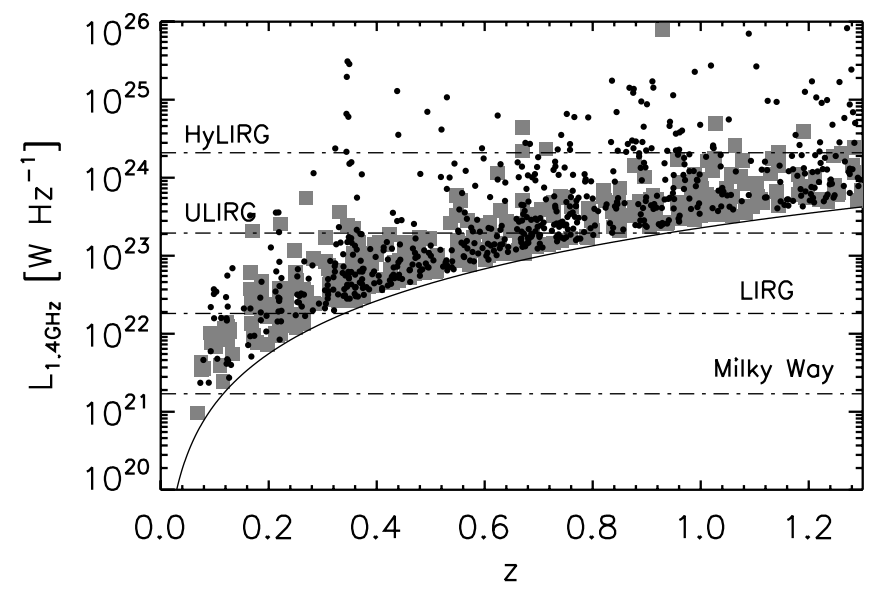

FIG. 16.- The $1.4 \mathrm{GHz}$ luminosity as a function of redshift for the 340 selected SF galaxies (gray squares) and 601 AGNs (black dots). The horizontal (dot-dashed) lines correspond to $1.4 \mathrm{GHz}$ luminosities typical for various classes of galaxies, obtained using the total IR-radio correlation (Bell 2003). The solid curved line corresponds to the VLA-COSMOS $5 \sigma$ limit of $\sim 50 \mu \mathrm{Jy}$. Note also that the VLA-COSMOS survey is sampling the entire LIRG and ULIRG populations out to redshifts of $\sim 0.4$ and $\sim 1$, respectively.

in particular the relative number of SF galaxies compared to AGNs rises at higher redshifts $(z \sim 1)$. This may be explained by the relatively high number density of ULIRGs expected at these redshifts (Le Floc'h et al. 2005; Caputi et al. 2007) in conjunction with the VLA-COSMOS detection limit, which at these redshifts allows one to sample only radio luminosities larger than $3 \times 10^{23} \mathrm{~W} \mathrm{~Hz}^{-1}$ (see Fig. 16). Furthermore, as the comoving volume surveyed at $z \sim 1$ is larger than that surveyed locally, the probability of detecting a ULIRG is also increasing at these redshifts. Effects of cosmic variance as a function of redshift cannot be excluded; however, they should be smaller than those of other deep radio surveys that typically probe significantly smaller areas. An increase of the AGN fraction at $z \sim 0.7$ is discernible. It possibly arises due to the dense large-scale structure component in the COSMOS field at this redshift (Scoville et al. 2007b; Guzzo et al. 2007).

In the bottom panel in Figure 15 we show the fractional contribution of the SF and AGN galaxies to the $z \leq 1.3$ matched radio population, as a function of redshift. On average, we find that the mean fractional contribution of SF and AGN galaxies to the $z \leq 1.3$ matched radio population is $34 \% \pm 14 \%$ and $66 \% \pm$ $14 \%$, respectively. This is strikingly similar to the relative numbers of SF and AGN galaxies in the local universe. Namely, if we apply the adopted $P 1$ color cut to the SDSS/NVSS galaxy sample (see $\S 4.1$ ), we find that $\sim 32 \%$ of the galaxies are star forming, and $\sim 68 \%$ are AGNs. If, as shown by the tests described in $\S 4.1$, our rest-frame color-based classification method can also reliably be applied to high-redshift galaxies, then the similarity of the SF and AGN fractions suggests that the two populations have similar evolutionary properties out to $z \sim 1.3$.

In Figure 16 we show the $1.4 \mathrm{GHz}$ luminosity as a function of redshift for the selected SF and AGN galaxies out to $z=1.3$. We also indicate the expected luminosity ranges for Milky Way-type galaxies, LIRGs, ULIRGs, and HyLIRGs, which were derived using the total IR-radio correlation (Bell 2003). It is noteworthy that the majority of galaxies with luminosities typical for HyLIRGs $\left(L_{\mathrm{IR}}>10^{13} L_{\odot}\right)$ was classified by the rest-frame color-based classification method as AGNs, consistent with the expected properties of these galaxies (e.g., Veilleux et al. 1999; Tran et al. 2001). This point is seen more clearly in Figure 17, where we show the distribution of the $1.4 \mathrm{GHz}$ luminosity for the selected SF and
AGN galaxies. The median luminosities are $\sim 1.6 \times 10^{23}$ and $\sim 3.2 \times 10^{23} \mathrm{~W} \mathrm{~Hz}^{-1}$ for SF and AGN galaxies, respectively. Although the median luminosities of the two populations are different only by a factor of 2 (note that this is drawn from a luminosity distribution of a flux-limited sample), there are some significant differences at both high and low radio luminosity. At high luminosities there is the strong decline of the number of SF galaxies with luminosities above $\sim 10^{24} \mathrm{~W} \mathrm{~Hz}^{-1}$, while AGNs show an extended tail toward the brightest $1.4 \mathrm{GHz}$ luminosities. Such a behavior is consistent with results from local studies, which suggested that "normal" 22 galaxies tend to have $L_{1.4 \mathrm{GHz}} \lesssim 10^{24} \mathrm{~W} \mathrm{~Hz}^{-1}$ (e.g., Condon 1992; Georgakakis et al. 1999). It is noteworthy that our SF and AGN galaxies were identified completely independently from their radio luminosity, yet their $1.4 \mathrm{GHz}$ luminosities match the expectations based on local studies. At low luminosity ( $\leqslant 2 \times 10^{22} \mathrm{~W} \mathrm{~Hz}^{-1}$; below the typical LIRG radio luminosity) the fraction of SF galaxies increases and the numbers of SF and AGN galaxies are similar to each other.

Furthermore, the luminosity distribution shown in Figure 17 agrees well with local results, which have shown that $L_{1.4 \mathrm{GHz}}$ for SF and (absorption- and emission-line) AGN galaxies shows overlapping distributions, and consequently no clear separation (e.g., Sadler et al. 1999; Georgakakis et al. 1999; Jackson \& Londish 2000; Chan et al. 2004). In the local universe Sadler et al. (1999) inferred a median $L_{1.4 \mathrm{GHz}}$ for $\mathrm{SF}$ and AGN galaxies to be $\sim 3 \times$ $10^{22}$ and $\sim 3 \times 10^{23} \mathrm{~W} \mathrm{~Hz}^{-1}$, respectively. Hence, our median value for the luminosity of VLA-COSMOS AGNs $\left(3.2 \times 10^{23} \mathrm{~W} \mathrm{~Hz}^{-1}\right)$ out to $z=1.3$ matches that inferred locally; however, for SF galaxies $\left(1.6 \times 10^{23} \mathrm{~W} \mathrm{~Hz}^{-1}\right)$ it is higher than that derived by Sadler et al. (1999). The latter is easily understood as the combined effect of the higher median redshift $(\sim 0.7)$ of the galaxies in our flux-limited sample (thus, not probing low $L_{1.4 \mathrm{GHz}}$ ) and of the higher level of star formation activity, which is observed going from redshift 0 to 1 (Madau et al. 1996; Hopkins 2004), also implying higher $L_{1.4 \mathrm{GHz}}$ (Condon 1992; Bell 2003).

We caution at this point that luminosity distributions of fluxlimited samples are strongly dependent on the detection limits, and their interpretation has to be approached carefully. For this reason, in Figure 17 we also show the luminosity distributions for volume-limited samples of our selected SF and AGN galaxies in three redshift ranges. On average, AGN galaxies have higher 1.4 GHz luminosities than SF galaxies, reassuring the validity of our selection method. Note, however, that in the lowest redshift range, the AGN and SF galaxy distributions are very similar. This is not surprising, but rather consistent with the observed luminosity range, which encompasses the region of indistinguishable space density of SF and AGN galaxies in the local universe (see local $1.4 \mathrm{GHz}$ luminosity functions for SF and AGN galaxies; e.g., Best 2004).

\subsection{The Population Mix in the VLA-COSMOS Survey}

In this section we study the contribution of different subpopulations to the total submillijansky radio population. The key question we want to answer is: is the submillijansky population dominated by any particular subpopulation, which may be the main cause for the observed flattening of the differential radio source counts below $1 \mathrm{mJy}$ (for VLA-COSMOS source counts; see Bondi et al. 2007)?

22 "Normal" galaxies, in terms of radio properties, are broadly defined as galaxies whose radio emission is not powered by a supermassive black hole. These galaxies are a mix of spiral, dwarf irregular galaxies, peculiar and interacting systems, as well as E/S0 galaxies with ongoing star formation (see Condon 1992 for a review). 

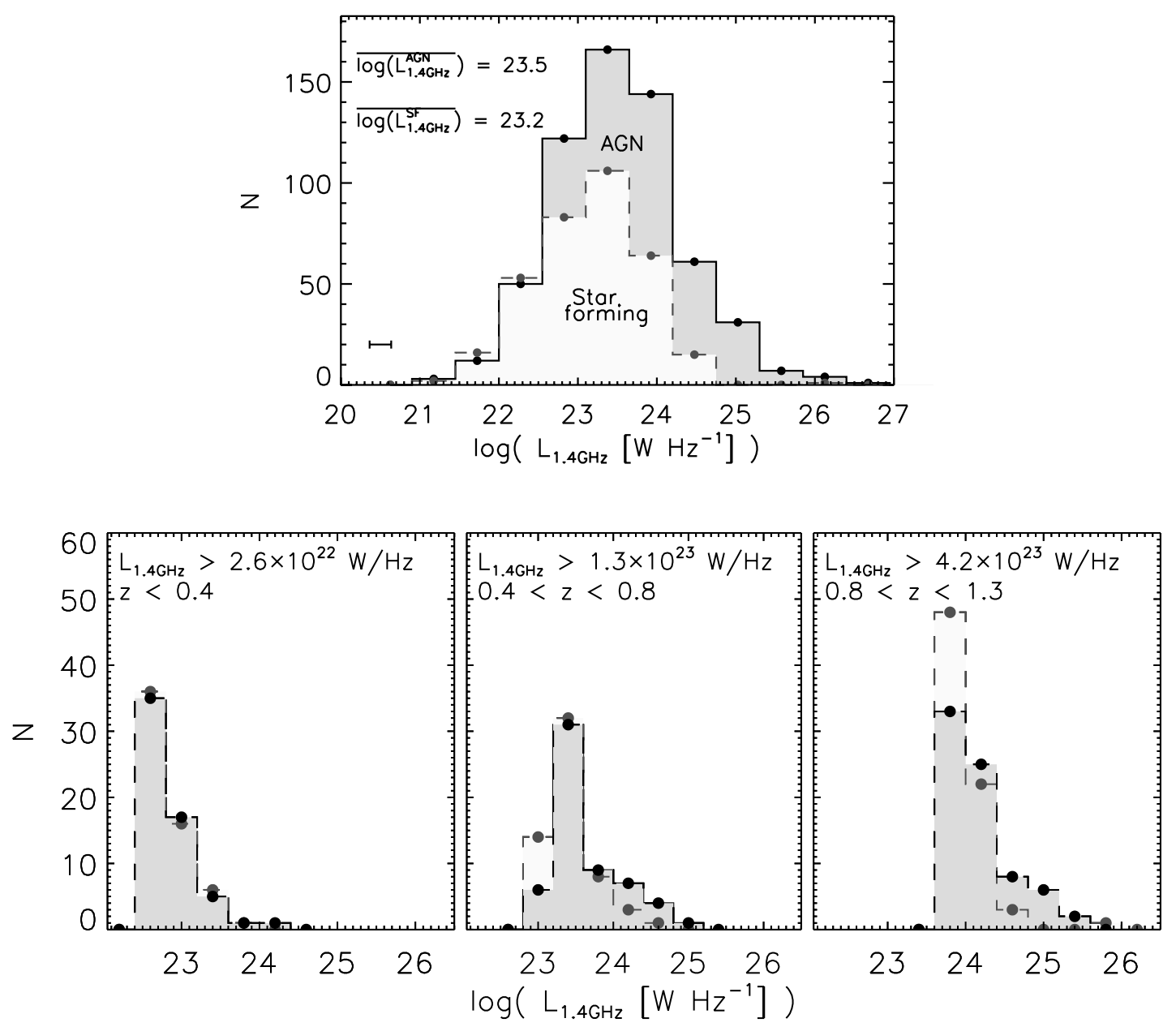

FIG. 17.-Distribution of the logarithm of the $1.4 \mathrm{GHz}$ luminosity for the selected SF (light gray shaded histograms) and AGN (dark gray shaded histograms) galaxies for the entire flux-limited sample (top), and for volume-limited samples in three redshift ranges (bottom panels). In the top panel the median log $\left(L_{1.4 \mathrm{GHz}}\right)$ values for the full SF and AGN samples, and the average error in $\log \left(L_{1.4 \mathrm{GHz}}\right)$ are indicated. The redshift and luminosity ranges of the volume-limited samples are given in the bottom three panels.

\subsubsection{Is the Matched Radio Sample at Submillijansky Levels Dominated by SF Galaxies?}

In order to obtain an insight into the 'population mix' of the faint radio sources in the matched radio source sample, in Figure 18 we show the distribution of the $1.4 \mathrm{GHz}$ total flux density, $F_{\text {tot }}$, for the $\mathrm{SF}$ and AGN galaxies in the matched radio sample out to $z=1.3$, as well as for the identified QSOs. ${ }^{23}$ Note that the remaining $z>1.3$ galaxies in the matched radio sample are defined as high-redshift (high-z) galaxies. The flux bins in Figure 18 are $0.15 \mathrm{mJy}$ wide. Such wide bins allow us to study the average behavior of the galaxies in the submillijansky population with decreasing flux densities. Our main aim is to answer one of the more controversial questions in radio astronomy: Is the submillijansky radio population dominated by SF galaxies or any other distinct subpopulation?

From the top panel in Figure 18, it is obvious that at flux densities above $\sim 0.7 \mathrm{mJy}$ we are hampered by low number statistics (the total number of sources in each bin is below 20). Therefore, for the purpose of this paper we focus only on sources with flux densities below $0.7 \mathrm{mJy}$ down to the VLA-COSMOS $5 \sigma$ limit of $\sim 50 \mu \mathrm{Jy}$.

The middle panel in Figure 18 shows the fractional distribution of the identified populations in the matched radio sample, with indicated Poisson errors. Our findings are as follows. QSOs

\footnotetext{
${ }^{23}$ Photometric redshift information for QSOs in the COSMOS survey is not available at this point.
}

contribute to the matched radio sample at a constant level of about $10 \%$. AGN galaxies below $z=1.3$ show a decreasing trend with decreasing flux densities, with their contribution to the matched radio sample dropping from $\sim 60 \%$ to less than $40 \%$. The SF galaxies at $z \leq 1.3$ in the flux density range of $50 \mu \mathrm{Jy}$ to $0.7 \mathrm{mJy}$ contribute fairly constantly at the given flux densities, with an average contribution of about $20 \%$. Note that the possible increment from $0.7 \mathrm{mJy}$ to $50 \mu \mathrm{Jy}$ of only $\sim 10 \%$ is not significant. In $\S 4.2 .2$ we have inferred that the photometric errors in the synthesized $P 1$ color introduce a positive bias of $\sim 5 \%$ for SF galaxies. As this bin contains the lowest number of SF galaxies, it is the most affected by this bias. In $\S 4.1 .2$, based on the local SDSS/NVSS sample of galaxies, we have shown that our restframe color-based classification method selects $\sim 70 \%$ of "real" SF galaxies, which make up $\sim 85 \%$ of the complete sample of SF galaxies, while the photometrically selected AGN sample is contaminated by SF galaxies at the $5 \%$ level. Assuming that the percentages of completeness and contamination, derived from the analysis of the SDSS/NVSS sample of galaxies, can also be safely applied to our VLA-COSMOS sample, we can use them to correct the observed fractions. Even with the correction the fractional contribution of SF galaxies at $z<1.3$ in the matched radio population essentially stays the same, i.e., about $\sim 20 \%$ at flux densities in the range from $50 \mu \mathrm{Jy}$ to $0.7 \mathrm{mJy}$. Contrary to previous studies (e.g., Seymour et al. 2004; Benn et al. 1993), our results show that SF galaxies at intermediate redshifts are not the dominant 

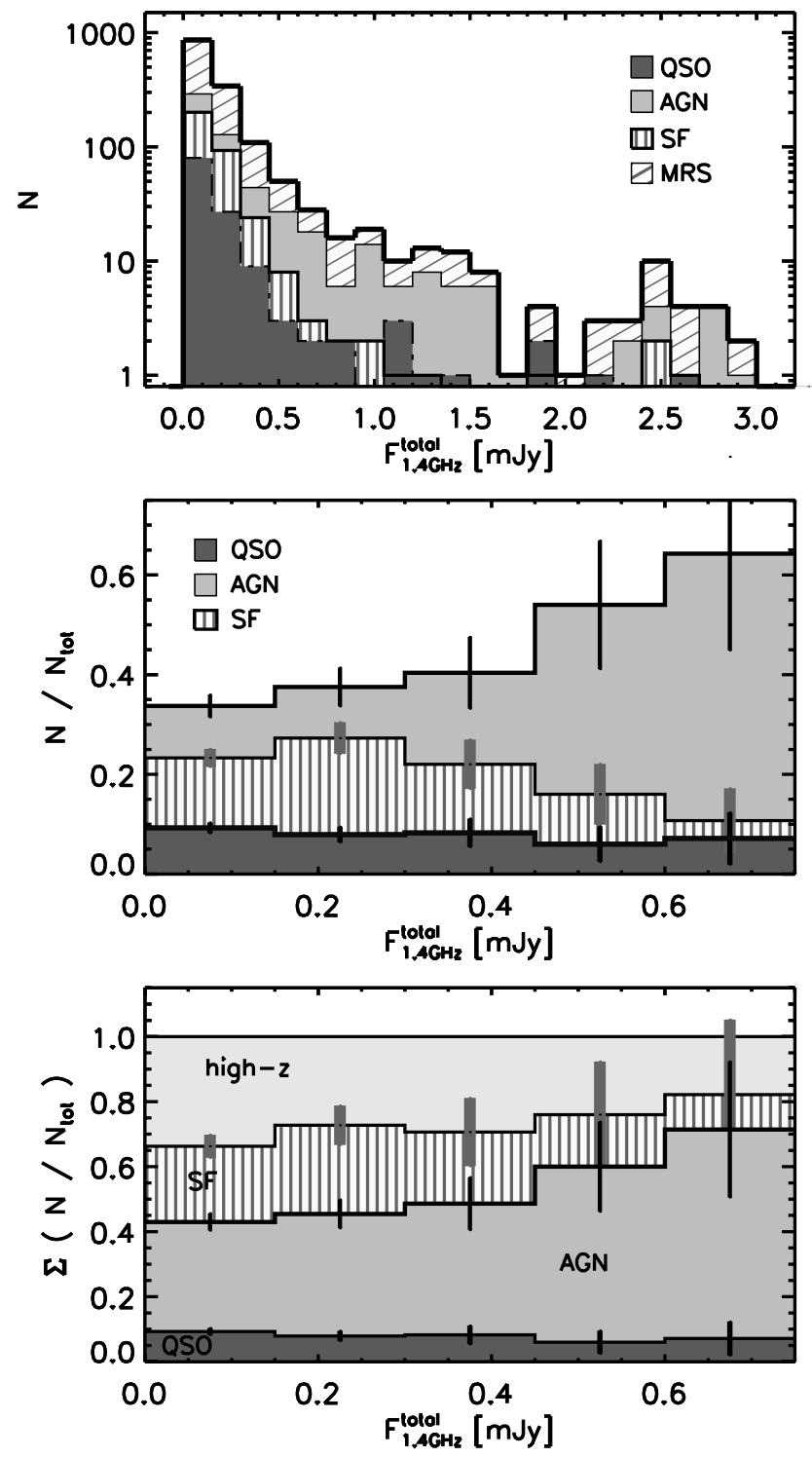

FIG. 18.-Top: Distribution of the $1.4 \mathrm{GHz}$ total flux for the SF (vertically hatched histogram) and AGN galaxies (light gray shaded histogram) in the matched radio sample out to $z=1.3$ (see also Fig. 17). Shown is also the distribution for the selected QSOs (dark gray shaded histogram), for which good redshift information is not available at this point. "MRS" stands for the matched radio sample. Middle: Relative contribution of SF, AGN galaxies out to $z=1.3$, and QSOs to the matched radio sample. Note that the missing fraction of sources consists of the high-redshift (high-z) objects, which we define as galaxies in our matched radio sample with $z>1.3$. Bottom: Cumulative distribution of SF, AGN galaxies out to $z=1.3$, QSOs, and high- $z$ galaxies in the matched radio sample. The indicated error bars in the middle and bottom panels are derived from Poisson statistics. Note also the different flux scales in the top and the middle/bottom panels.

population at submillijansky flux density levels. However, it may be possible that a significant number of SF galaxies at $z>1.3$ exists, which may contribute strongly to the submillijansky population. We investigate this possibility in the bottom panel in Figure 18 and in Figure 19 and show below that this is not the case.

About $30 \%$ of the matched radio sample consists of galaxies at redshifts beyond $z=1.3$ (high-z; see bottom panel in Fig. 18). The contribution of these galaxies marginally increases from $20 \%$ at $0.7 \mathrm{mJy}$ to $35 \%$ at $50 \mu \mathrm{Jy}$. Although we were not able to apply our rest-frame color-based classification method to these galaxies in order to classify them, we can nevertheless draw some conclusion about their nature by studying their multiwavelength properties. Therefore, in Figure 19 we plot the MIR diagnostic

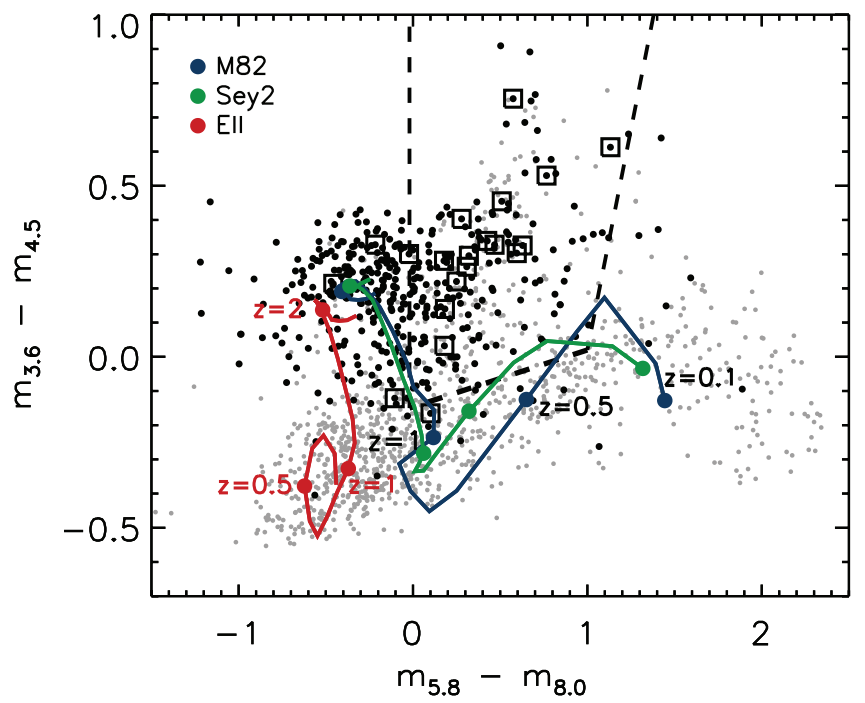

FIG. 19.-MIR color-color diagram (analogous to Fig. 10, bottom) for the full matched radio sample. Gray dots represent sources classified using our classification method (i.e., SF and AGN galaxies out to $z=1.3$, and QSOs), while the black dots show the high- $z$ sources (galaxies in the matched radio sample beyond $z=1.3$ ). Open black squares represent the high- $z$ sources that have an XMMNewton point source counterpart. The dashed lines are indicated to guide the eye, and correspond to the AGN type 1 selection region, proposed by Stern et al. (2005). The curved green, blue, and red lines correspond to the color-color tracks, obtained from SEDs of the starburst galaxy M82, a composite of 28 Seyfert 2 galaxies, and a 13 Gyr old elliptical galaxy, respectively, in the redshift range from 0.1 to 2.5 .

diagram for the high-z galaxies and compare their properties to the classified sources in the matched radio sample. We overlay nonevolving color tracks for the typical starburst galaxy M82, an elliptical 13 Gyr old galaxy, and a Seyfert type 2 SED, obtained from a composite spectrum of 28 randomly chosen Seyfert galaxies (Polletta et al. 2007). As expected, the properties of high- $z$ galaxies are consistent with properties of higher redshift $(z>$ $1.5)$ galaxies. However, the region they occupy in this MIR colorcolor diagram is occupied in a similar way by different subpopulations, such as SF, Seyfert-type, and passively evolving galaxies, in the redshift range from about 1.5 to 3 (see Fig. 19, color tracks). Furthermore, it is possible that a fraction of these sources are broad-line AGNs as they are located within the area outlined by dashed lines, which was proposed by Stern et al. (2005) for the selection of AGN type 1 (see also Caputi et al. 2007). This is further strengthened by the 22 high- $z$ sources which were also identified as X-ray point sources and lie within the type 1 AGN selection region. Furthermore, given the high redshift of these sources, combined with the XMM-Newton detection limit, it is highly unlikely that any of these sources may be star forming (see, e.g., Fig. 14 in Trump et al. 2007). This analysis alone already indicates that the matched radio sources beyond $z=1.3$ are most probably a mixture of different subpopulations.

The population mix in the high- $z$ sample is further affirmed by the distribution of the high- $z$ galaxies in the $B z K$ diagram (shown in Fig. 20), which is commonly used for the selection of $z>1.4$ galaxies, and separates well passively evolving galaxies from those originally defined as "star forming" at $z>1.4$ (Daddi et al. 2004). From Figure 20 it becomes obvious that the $B z K$ criterion does not select a pure SF sample at $z>1.4$, as initially postulated, but a sample that comprises both SF galaxies and low-luminosity AGNs. In addition, the X-ray-detected high- $z$ sources, which may be classified as AGNs with high confidence as discussed above, also lie within this region. Therefore, the SED color tracks combined with the distribution of the high-z galaxies (Fig. 20, black dots), 


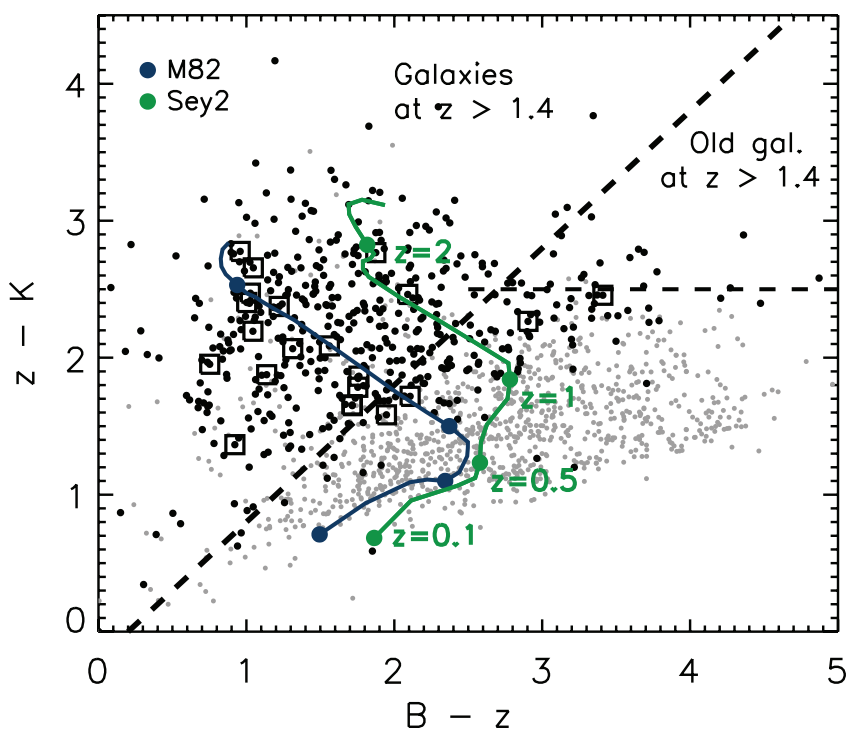

FIG. 20.-The $B-z$ vs. $z-K$ color-color diagram for the entire matched radio sample. The symbols are analogous to Fig. 19; gray dots represent sources identified using our classification method (i.e., SF and AGN galaxies out to $z=1.3$ and QSOs), the black dots show the high- $z$ sources, and open squares show XMM-Newton-detected point sources in the high- $z$ sample. The dashed lines separate regions of passively evolving (top right; outlined by the diagonal and horizontal dashed lines) and SF galaxies (left of the diagonal dashed line) at $z>1.4$, adopted from Daddi et al. (2004). The curved green and blue lines correspond to the color-color tracks, obtained from SEDs of the starburst galaxy M82, and a Seyfert 2 composite, respectively, in the redshift range from 0.1 to 2.5. Note that Seyfert-type galaxies at $z>1.4$ are also present in the region of "SF galaxies," as initially postulated by Daddi et al. (2004)

and the X-ray-detected subsample suggest that our $z>1.3$ matched sources are a fair mixture of SF and AGN galaxies, yielding that the high- $z$ galaxies continue to consist of different galaxy populations at higher redshifts.

For reference, the $i$-band magnitude distribution for the identified subsamples in the matched radio sample is shown in Figure 21. Note that the high- $z$ galaxies are the faintest optical sources, consistent with the expectations for high-redshift sources drawn from a flux-limited sample. However, we want to mention that even in the most extreme case in which all of the high- $z$ galaxies were star forming, still the fractional contribution of SF galaxies to the matched radio source population would not dominate over the contribution of AGNs and QSOs, but the two contributions would be rather comparable.

As shown in Figure 20 it is likely that both classes of objects contribute similarly to the high- $z$ galaxies, although their exact relative fractions cannot be determined from this analysis. However, if we assume that roughly $50 \%$ of the high- $z$ galaxies are $\mathrm{SF}$ galaxies, then the fraction of SF galaxies in the matched radio sample would increase to less than $40 \%$. Therefore, we conclude that the population of SF galaxies is not the dominant population at submillijansky levels, at least in our sample of radio sources with optical counterparts out to $i=26$.

Our results are strikingly similar to the recent results by Padovani et al. (2007), who studied the submillijansky radio source population in the Chandra Deep Field-South down to a $5 \sigma$ limit of $42 \mu \mathrm{Jy}$. They found that SF galaxies make up only about $20 \%-45 \%$ (i.e., roughly $1 / 3$ ) of the submillijansky radio sources.

7.2.2. The Contribution to the Submillijansky Radio Population from Radio Sources with No, or with Flagged, Optical Counterparts

Most of the previous studies (as well as our study up to this point) of the faint radio population have relied on subsamples

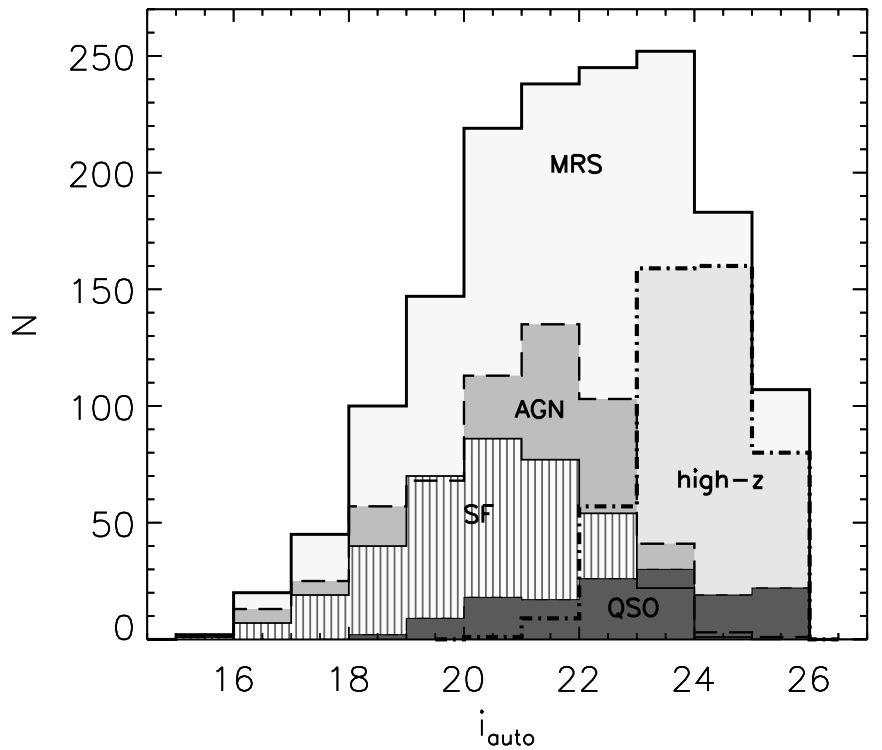

FIG. 21.-Distribution of the $i$-band magnitude (Subaru where available, otherwise CFHT) for sources in the VLA-COSMOS matched radio sample (MRS). Also shown are the distributions for the identified subsamples of sources: QSOs, $\mathrm{SF}$ (SF), AGN, and high-z galaxies

of the observed radio sources, which have been identified with optical counterparts out to $i=26$, as representative of the entire submillijansky radio population. For example, Benn et al. (1993) used a sample of only 87 out of 523 (i.e., less than $20 \%$ ) $1.4 \mathrm{GHz}$ radio sources above $0.1 \mathrm{mJy}$, for which they obtained optical spectroscopy $(B<22)$, to conclude that above $1 \mathrm{mJy}$ about $50 \%$ of the galaxies were SF or Seyfert galaxies, while below $1 \mathrm{mJy}$ the fraction increases to $\sim 90 \%$. Furthermore, Gruppioni et al. (1999) studied optical spectroscopic properties of 34 radio sources above $0.2 \mathrm{mJy}$ in the Marano Field down to $B=24$. This sample comprised $\sim 60 \%$ of the entire sample of faint radio sources, and they concluded that the SF galaxies do not constitute the main galaxy population of their radio sources, and even at submillijansky levels the majority of their radio sources were identified with earlytype galaxies, consistent with AGNs. Gruppioni et al. attributed the difference in their results compared to the results from Benn et al. (1993) to the fainter optical magnitude limit reached for their radio sample. In this work we have an order of magnitude larger sample size (1558 sources with optical counterparts) and a significantly deeper optical limit $(i=26)$ than previous studies. However, our matched radio source sample still consists of only $\sim 65 \%$ of the VLA-COSMOS $1.4 \mathrm{GHz}$ sources. Therefore, it is important to investigate the contribution of the remaining $\sim 35 \%$ of the radio sources with no identified or flagged optical counterparts to the submillijansky population. It may indeed be possible that a missing population of objects, which significantly contributes to the submillijansky population, is hidden in this sample. We show below that this is not the case.

If the above hypothesis is true, then the properties (such as, e.g., MIR colors) of these remaining objects are expected to be distinct from the properties of objects in the matched radio sample. In order to shed light on this, in Figure 22 (top) we show the distribution of the total flux density for the 1558 sources in the matched radio sample, and for the remaining 830 sources that were (1) not identified with optical counterparts with $i \leq 26$ or (2) have optical counterparts with $i \leq 26$ but in a photometrically masked-out region (see $\S 3.1$ ). The bottom panel shows the fractional contribution of these two samples compared to the entire VLA-COSMOS 1.4 GHz population. The fraction of matched 

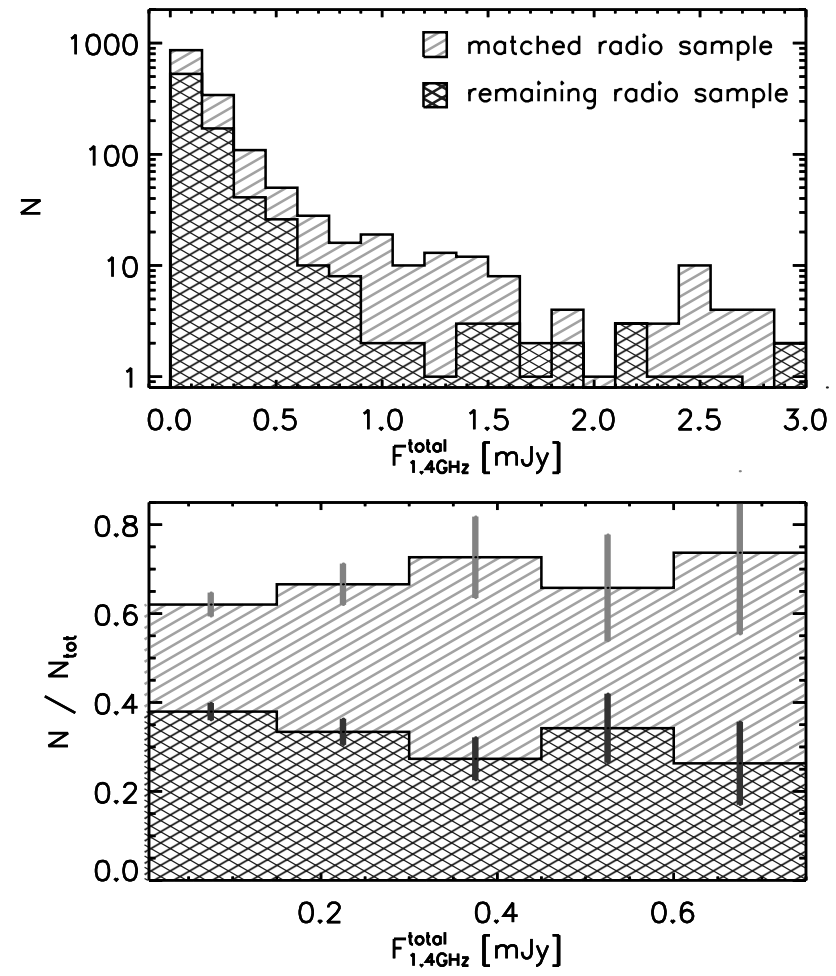

FIG. 22.-Top: Distribution of the $1.4 \mathrm{GHz}$ total flux for sources in the matched radio sample (hatched histogram), and for other VLA-COSMOS sources that have either no identified or a photometrically flagged, optical counterpart brighter than $i=26$ (cross-hatched histogram). Bottom: Fractional distribution of the two samples compared to the entire sample of $1.4 \mathrm{GHz}$ radio sources. Indicated error bars are derived from Poisson statistics.

radio sources is statistically consistent to be constant at $\sim 65 \%$ at all faint flux density levels, although formally it decreased from $\sim 75 \%$ at $\sim 0.7 \mathrm{mJy}$ to $\sim 60 \%$ at the limit of the VLA-COSMOS survey. On the basis of these high percentages, it is unlikely that any further population, which is not present in our matched radio sample, could account for a significant fraction of the submillijansky population.

This is further strengthened by the distribution of the remaining sources in the MIR color-color diagram, shown in Figure 23, which is consistent with the expected distribution for a mixed sample of SF and AGN galaxies and QSOs at all redshifts in the range from the local to the highest observable redshifts. An additional affirmation of the mix of the population arises from the 31 sources that have been detected as X-ray point sources (see Fig. 23). It is worth noting, however, that $\sim 60 \%$ of these sources are consistent with higher redshift objects $(z \gtrsim 1.3)$, while this is true only for $\sim 50 \%$ of the galaxies in the matched radio sample (see Fig. 19). We therefore conclude that the radio sources without identified or with flagged optical counterparts brighter than $i=26$ most likely comprise a mixture of different source populations (SF, AGN, and QSO), similar to although on average at a higher redshift than the radio sources in our matched radio sample. Furthermore, the relatively small total percentage of these sources cannot significantly alter the results about the population mix in the $1.4 \mathrm{GHz}$ VLA-COSMOS radio sample, inferred in the previous section.

\subsection{Concluding Remarks on the Composition of the Faint Radio Population}

The faint submillijansky radio population comprises the radio population responsible for the upturn of the differential radio

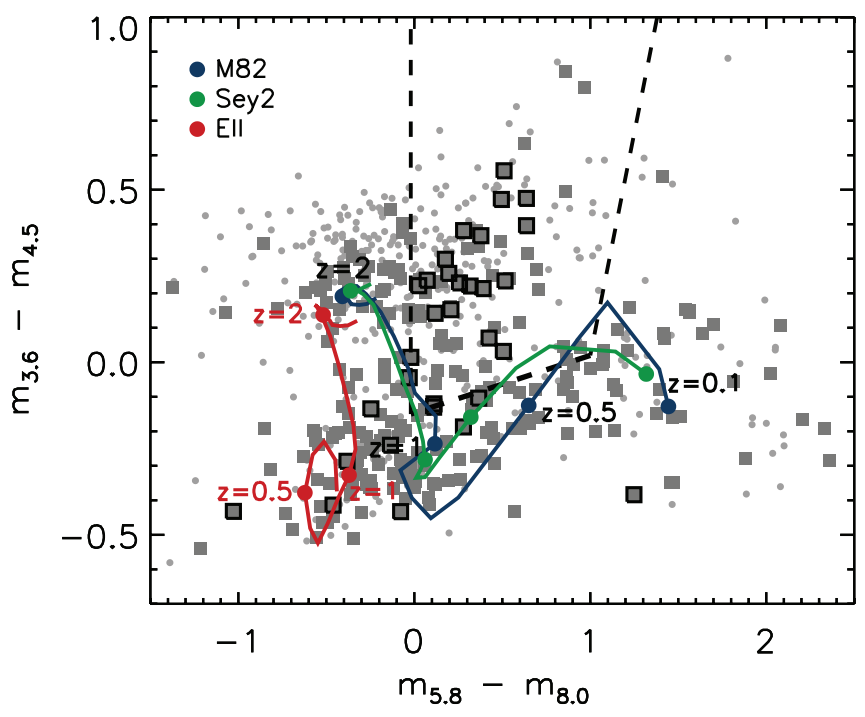

FIG. 23.- Same as Fig. 19, but for 813 VLA-COSMOS $1.4 \mathrm{GHz}$ sources (gray dots) that have either no identified or a photometrically flagged optical counterpart brighter than $i=26$, but that have IRAC detections (i.e., $\sim 75 \%$; see $\S 3.4)$. Gray squares indicate the sources that have an optical counterpart with $i \leq 26$, but that is within a masked-out area. Black open squares represent the sources that have $X M M$-Newton point-source detections. The clumping of objects in the top left quadrant is consistent with SF, Seyfert, and passive galaxies in the redshift range from about 1.5 to 3 , suggesting that over $\sim 60 \%$ of the VLACOSMOS “remaining” sources are high-redshift galaxies or QSOs.

source counts below $1 \mathrm{mJy}$ (see, e.g., Bondi et al. 2007), and it has been the subject of many studies, and a matter of great debate in the past three decades (Condon 1984; Windhorst et al. 1985; Gruppioni et al. 1999; Seymour et al. 2004; Simpson et al. 2006). This radio population has been interpreted as a new rising population of objects, which do not significantly contribute at higher radio flux density levels. However, results from studies that tried to reveal the exact composition of the submillijansky population have been highly discrepant. It was suggested that the majority of this faint radio population consists of faint blue galaxies, and it was assumed that these galaxies are undergoing significant star formation (Windhorst et al. 1985). The spectroscopic study by Benn et al. (1993) although analyzing only less than $20 \%$ of their radio sample, supported this result indicating that the fraction of $\mathrm{SF}$ and Seyfert galaxies rises from about $50 \%$ to $90 \%$ in the range from super- to submillijansky flux densities. However, Gruppioni et al. (1999), who performed a deeper optical spectroscopic analysis of a larger fraction $(\sim 60 \%)$ of their radio sources, disagreed with this result, identifying the majority of their submillijansky radio sources with early-type galaxies, in which the radio emission is produced by AGNs. Furthermore, Georgakakis et al. (1999), who obtained optical spectroscopy for $\sim 40 \%$ of their radio sample, identified roughly $50 \%$ SF galaxies in the submillyjansky population in the Phoenix Deep survey (this fraction has been supported by the spectroscopy presented in Afonso et al. 2005). Recently, using a combination of optical and radio morphology as an identifier for AGN and SF galaxies on $\sim 90 \%$ of their radio sources in the SSA13 field, Fomalont et al. (2006) suggested that less than $\sim 40 \%$ of the radio sources below 1 mJy are AGNs. On the other hand, Padovani et al. (2007) based their SF/AGN classification on a combination of optical morphologies, X-ray luminosities, and radio-to-optical flux ratios of their radio sources in the CDFS (Chandra Deep Field-South) and indicated that only about $20 \%-40 \%$ of the faint radio sources are made up of SF galaxies.

As already mentioned in the $\S 1$ there are two main reasons for such controversial results in the past literature: (1) the identification 
fraction of radio sources with optical counterparts spans a wide range $(20 \%-90 \%)$ in samples from radio deep fields, and (2) the methods for the separation of SF from AGN galaxies have been highly heterogeneous. Furthermore, the first and second point seem to exclude each other. Namely, the most efficient separation methods (e.g., intermediate- to high-resolution optical spectroscopy) generally led to a small identification fraction, whereas large radio samples with high optical identification fractions usually lacked robust $\mathrm{SF} / \mathrm{AGN}$ indicators. In this paper we have tried to make a strong conjunction of these two points. We introduced a new method of separating SF from AGN galaxies that relies strongly on rest-frame color properties and, thus, allowed us to perform a robust classification of $\sim 65 \%(=1558)$ of the $1.4 \mathrm{GHz}$ VLA-COSMOS radio sources down to $\sim 50 \mu \mathrm{Jy}$ with identified optical counterparts out to $i=26$ without the need for, e.g., optical spectroscopy. Thus, we managed to reach both a high identification fraction and a robust source classification. However, in order to avoid any possible biases, we also used the full panchromatic COSMOS data set to put constraints on the properties of the remaining $\sim 35 \%$ of the radio sources that were not identified with optical counterparts with $i \leq 26$, or that have optical counterparts with $i \leq 26$ but with uncertain photometry due to blending and saturation in the optical images. In short, we have obtained a complete view of the population mix of the submillijansky radio sources, using the largest sample to date of 2388 faint $(5 \sigma \approx$ $50 \mu \mathrm{Jy}$ ) radio sources at $1.4 \mathrm{GHz}$. Our combined methods allow us to reveal the true nature of the submillijansky radio sources in the VLA-COSMOS survey on a sound statistical basis.

Jarvis \& Rawlings (2004) first suggested that the observed flattening of the differential radio source counts below $1 \mathrm{mJy}$ may be caused by radio-quiet AGNs, i.e., radio-quiet QSOs and type 2 AGNs (consistent with our AGN class), rather than SF galaxies. Making use of the observed correlation between $\mathrm{X}$-ray and radio luminosity and the empirically derived X-ray luminosity function, they computed the $1.4 \mathrm{GHz}$ luminosity function for radioquiet AGNs out to high redshifts and used it as the key ingredient to model the radio source counts at faint flux density levels (see Jarvis \& Rawlings 2004 and references therein). However, they did not take into account SF galaxies as an important population to explain the shape of the counts. Such an interpretation managed to represent the differential radio source counts well down to $\sim 250 \mu \mathrm{Jy}$, but failed at fainter flux densities. Our results, derived in the previous sections, show that indeed SF galaxies are not the dominant population at submillijansky levels in the range of $50 \mu \mathrm{Jy}$ to $0.7 \mathrm{Jy}$; however, they still contribute about $30 \%-$ $40 \%$ at these flux densities. Based on our large sample of 2388 VLA-COSMOS radio sources we find that our classified AGNs, which comprise mostly low-luminosity and type 2 AGNs, make up $50 \%-60 \%$ of the faint radio population with a decreasing trend toward fainter flux densities, while the identified QSOs, which are mostly type 1 AGNs, form a minor contribution of $\sim 10 \%$ of the submillijansky radio population in the range of $50 \mu \mathrm{Jy}$ to $0.7 \mathrm{Jy}$. Thus, our observational results show that the population mix in the faint radio population contains a fair contribution of both SF galaxies and (low-luminosity and obscured) AGNs, at least down to $50 \mu \mathrm{Jy}$. Thus, separate luminosity functions for both populations (see Cowie et al. 2004) must be taken into account in order to fully explain the flattening of the radio source counts below $1 \mathrm{mJy}$. The 1.4 GHz luminosity function for SF and AGN galaxies at intermediate redshift, as well as the modeling of the $1.4 \mathrm{GHz}$ radio source counts using these new observational constrains and further analyzes of the population mix in the VLA-COSMOS survey, is going to be fully addressed and presented in a number of up- coming publications ( Smolčić et al. 2008; P. Ciliegi et al. 2008, in preparation; T. Paglione et al. 2008, in preparation).

\section{SUMMARY}

Our newly developed rest-frame color-based classification method, in conjunction with the VLA-COSMOS $1.4 \mathrm{GHz}$ observations, enabled us to make, for the first time, a thorough distinction between sources where the $1.4 \mathrm{GHz}$ radio emission is predominantly driven by star formation processes from those where it is driven by SMBH processes, regardless of the luminosity of the latter, and apply it to currently the largest sample of $15581.4 \mathrm{GHz}$ sources, with optical counterparts brighter than $i_{\mathrm{AB} \leq 26}$, and complete down to $\sim 50 \mu \mathrm{Jy}$.

We have explored to full detail the composition of the submillijansky radio population making use of the entire sample of 2388 VLA-COSMOS radio sources detected above $5 \sigma(\approx 50 \mu \mathrm{Jy})$, in conjunction with the panchromatic (X-ray to radio) COSMOS data set. We find that SF galaxies are not the dominant population at submillijansky flux density levels, as believed previously, but that rather they make up an approximately constant fraction of $30 \%-40 \%$ in the flux density range of $\sim 50 \mu \mathrm{Jy}$ to $0.7 \mathrm{mJy}$. The radio population at these flux densities is a mixture of roughly $30 \%-40 \%$ SF and 50\%-60\% AGN galaxies, with a minor contribution $(\sim 10 \%)$ of QSOs.

The authors would like to thank M. Polletta for the spectral template library. V. S. would like to thank E. Bell, G. Fabbiano, G. Helou, and D. Frayer for insightful discussions, as well as M. Schartmann for his help regarding FORTAN compilers. C. C., E. S., and V. S. acknowledge support from NASA grant HSTGO-09822.31-A. C. C. would like to acknowledge support from the Max Planck Society and the Alexander von Humboldt Foundation through the Max-Planck-Forschungspreis 2005. K. J. acknowledges support by the German DFG under grant SCHI 536/3-1. C. J. W. is supported by the MAGPOP Marie Curie EU Research and Training Network.

This work is based on observations with the National Radio Astronomy Observatory, which is a facility of the National Science Foundation operated under cooperative agreement by Associated Universities, Inc., the NASA/ESA Hubble Space Telescope, obtained at the Space Telescope Science Institute, which is operated by AURA, Inc., under NASA contract NAS 05-26555, and XMMNewton, an ESA science mission with instruments and contributions directly funded by ESA Member States and NASA. This work is also based on data collected at the Subaru Telescope, which is operated by the National Astronomical Observatory of Japan, the European Southern Observatory, Chile, Kitt Peak National Observatory, Cerro Tololo Inter-American Observatory, and the National Optical Astronomy Observatory, which are operated by the Association of Universities for Research in Astronomy, Inc., (AURA) under cooperative agreement with the National Science Foundation. It is based also on observations obtained with MegaPrime/MegaCam, a joint project of CFHT and CEA/ DAPNIA, at the Canada-France-Hawaii Telescope (CFHT), which is operated by the National Research Council (NRC) of Canada, the Institut National des Science de l'Univers of the Centre National de la Recherche Scientifique (CNRS) of France, and the University of Hawaii. This work is based in part on data products produced at TERAPIX and the Canadian Astronomy Data Centre.

Funding for the Sloan Digital Sky Survey (SDSS) and SDSS-II has been provided by the Alfred P. Sloan Foundation, 
the Participating Institutions, the National Science Foundation, the US Department of Energy, the National Aeronautics and Space Administration, the Japanese Monbukagakusho, the Max Planck Society, and the Higher Education Funding Council for England. The SDSS Web site is http://www.sdss.org/.

The SDSS is managed by the Astrophysical Research Consortium (ARC) for the Participating Institutions. The Participating Institutions are the American Museum of Natural History, Astrophysical Institute Potsdam, University of Basel, University of Cambridge, Case Western Reserve University, the University of Chicago, Drexel University, Fermilab, the Institute for Advanced Study, the Japan Participation Group, the Johns Hopkins University, the Joint Institute for Nuclear Astrophysics, the Kavli Institute for Particle Astrophysics and Cosmology, the Korean Scientist Group, the Chinese Academy of Sciences (LAMOST), Los Alamos National Laboratory, the Max Planck Institute for Astronomy (MPIA), the Max Planck Institute for Astrophysics (MPA), New Mexico State University, Ohio State University, University of Pittsburgh, University of Portsmouth, Princeton University, the United States Naval Observatory, and the University of Washington.

\section{APPENDIX A}

\section{THE REST-FRAME COLORS $P 1$ AND $P 2$}

The rest-frame colors $P 1$ and $P 2$ are a linear superposition of colors in the wavelength range of 3500-5800 $\AA$, obtained using the modified Strömgren photometric system (Odell et al. 2002; Smolčić et al. 2006). ${ }^{24} P 1$ and $P 2$ are principal component axes optimally characterizing the galaxy locus in two-dimensional color-color space. $P 1$ measures the position along the galaxy locus, and $P 2$ measures the position perpendicular to it (see Fig. 4 in Smolčic et al. 2006). As the galaxy locus is slightly curved, the functional form of the restframe colors is given separately for the blue and red ends, with a boundary at $v z-y z=0.646$. Hence, for galaxies with $v z-y z \leq 0.646$ $(P 1, P 2)$ are given as

$$
\begin{gathered}
P 1=0.911\left(c_{1}-0.646\right)+0.412\left(c_{2}-0.261\right), \\
P 2=-0.412\left(c_{1}-0.646\right)+0.911\left(c_{2}-0.261\right),
\end{gathered}
$$

and for galaxies with $v z-y z>0.646$ as

$$
\begin{gathered}
P 1=0.952\left(c_{1}-0.646\right)+0.307\left(c_{2}-0.261\right), \\
P 2=-0.307\left(c_{1}-0.646\right)+0.952\left(c_{2}-0.261\right),
\end{gathered}
$$

where $c_{1}=v z-y z$ and $c_{2}=b z-y z$.

\section{APPENDIX B}

\section{DERIVATION OF COMPLETENESS AND CONTAMINATION OF THE PHOTOMETRICALLY SELECTED SAMPLES OF SF GALAXIES AND AGNs}

\section{B1. SDSS/NVSS GALAXY SAMPLE}

Given both the available spectroscopy and photometry of the local galaxy sample (SDSS/NVSS), we can easily access the completeness and contamination of the photometrically selected samples of SF and AGN galaxies as follows. In Figure 24 we show the differential and cumulative distributions of $P 1$ for the SDSS/NVSS SF, composite, and AGN galaxies. Here the classification is based on the BPT diagram for the galaxies with emission lines in their spectra, while all galaxies without emission lines are included in the AGN class. These "absorption-line AGNs" constitute the major fraction of all the AGNs $(\sim 80 \%)$ in the sample. The top panel shows the $P 1$ histograms for these three types of objects, normalized by the total number of objects. In the middle panels in Figure 24 we show the fractions for the SF, AGN and composite galaxies for the selection of SF (left) and AGN (right) galaxies. The fractions were computed in such a way that for each $P 1$ value $\left(P 1_{i}\right)$ the distributions were normalized to the total number of galaxies in the sample with $P 1 \leq P 1_{i}$ (for SF galaxies; left), or $P 1>P 1_{i}$ (for AGN galaxies; right). In this way, we obtain the fraction of SF, AGN and composite galaxies within the full sample that was selected only by applying a $P 1$ color cut. Furthermore, in the bottom panels in Figure 24 , we show the cumulative distributions of the SF (left) and AGN (right) galaxies, scaled to the total number of SF and AGN galaxies, respectively, thus showing their completeness as a function of $P 1$. Selecting galaxies with $P 1 \leq 0.15$, and defining them as the "photometrically selected sample of SF galaxies," leads to a sample that contains $\sim 20 \%$ AGNs, $\sim 10 \%$ composite objects, and $\sim 70 \%$ "real" (i.e., spectroscopically identified) SF galaxies (see Fig. 24, middle left). The latter make up $\sim 85 \%$ of all real SF galaxies (see Fig. 24 , bottom left). On the other hand, using the color cut to generate the photometrically selected AGN galaxy sample $(P 1>0.15)$ leads to a sample that contains $\sim 5 \%$ SF galaxies, $\sim 15 \%$ composite objects, and $\sim 80 \%$ real AGN galaxies (see Fig. 24 , middle right ), where the latter make up $\sim 90 \%$ of all real AGN galaxies (see Fig. 24, bottom right).

\section{B2. SDSS/NVSS/IRAS GALAXY SAMPLE}

The BPT diagram for $\sim 830$ SDSS/NVSS/IRAS emission-line galaxies is shown in Figure 25, color coded using the $(P 1, P 2)$ plane. A strong correlation between $P 1$ and $\log ([\mathrm{N}$ II $\lambda 6584] / \mathrm{H} \alpha)$, very similar to the correlation observed for the full sample, is discernible.

24 The filter response curves are available at http://www.mpia-hd.mpg.de/COSMOS. 

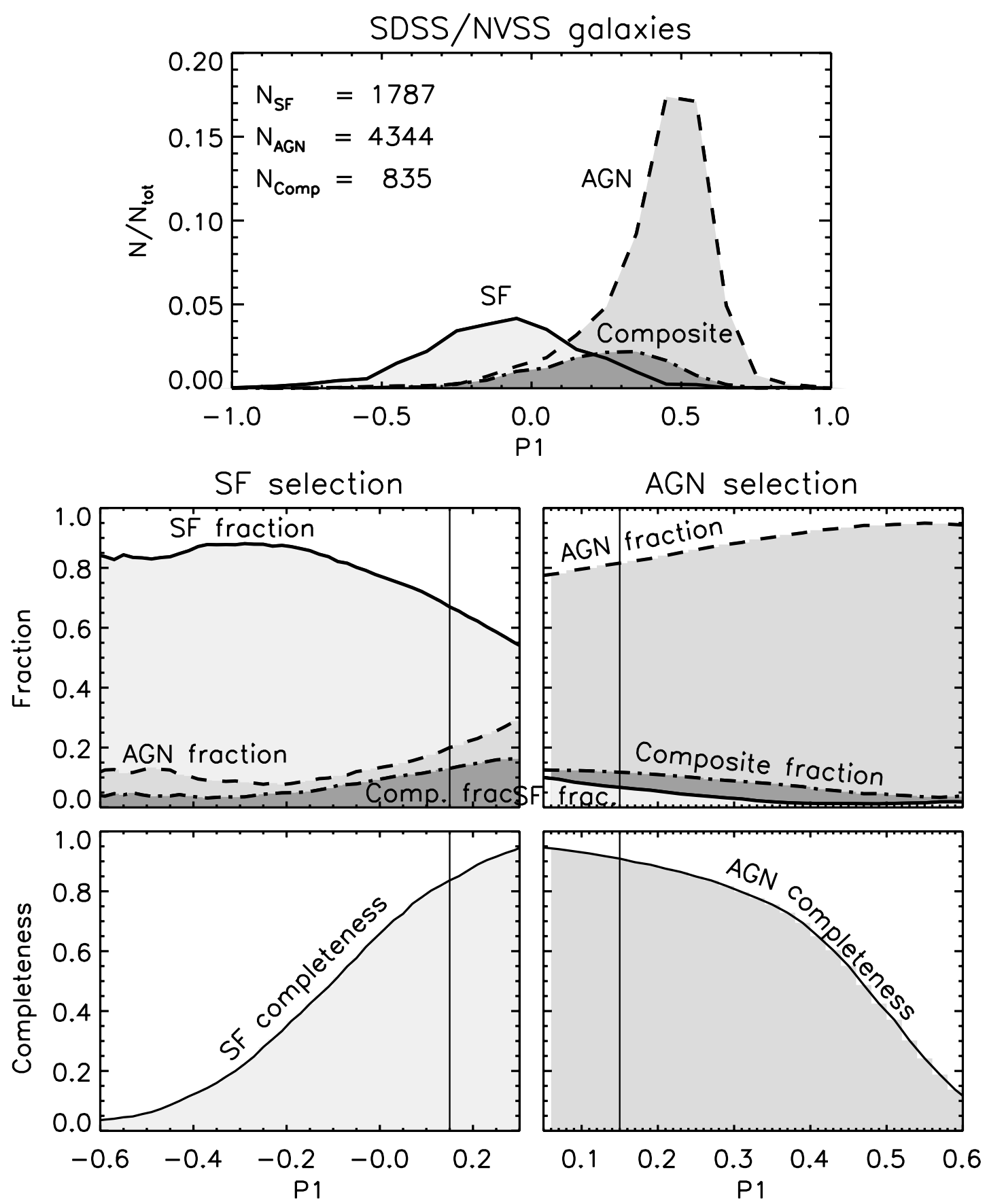

FIG. 24.-Differential and cumulative distributions of the rest-frame color $P 1$ for $\sim 7000$ SDSS galaxies, in the redshift range of 0.01 to 0.3 , drawn from the DR1 "main" spectroscopic sample, that have $1.4 \mathrm{GHz}$ NVSS detections. The galaxies were spectroscopically classified as SF (SF; thick line), AGN (dashed line), or composite (dot-dashed line; see text for details). The top panel shows the $P 1$ histograms for these three types of objects. The middle left panel shows the fraction of these three types of galaxies as a function of $P 1$. These distributions are normalized in such a way that for each $P 1$ value the fraction of the three types of galaxies within the total selected sample can be read off. The bottom left panel shows the cumulative distribution of $P 1$ for SF galaxies, thus showing to which completeness the "real" (i.e., spectroscopically classified) SF galaxies are selected for any given $P 1$ color-cut. The bottom and middle right panels are analogous to the bottom and middle left panels, but for AGN galaxies. Note also that the cumulative distributions for AGNs were computed as a function of decreasing $P 1$. The vertical (thin solid) lines in the middle and bottom panels designate the value of $P 1$, chosen to separate SF galaxies from AGNs. 

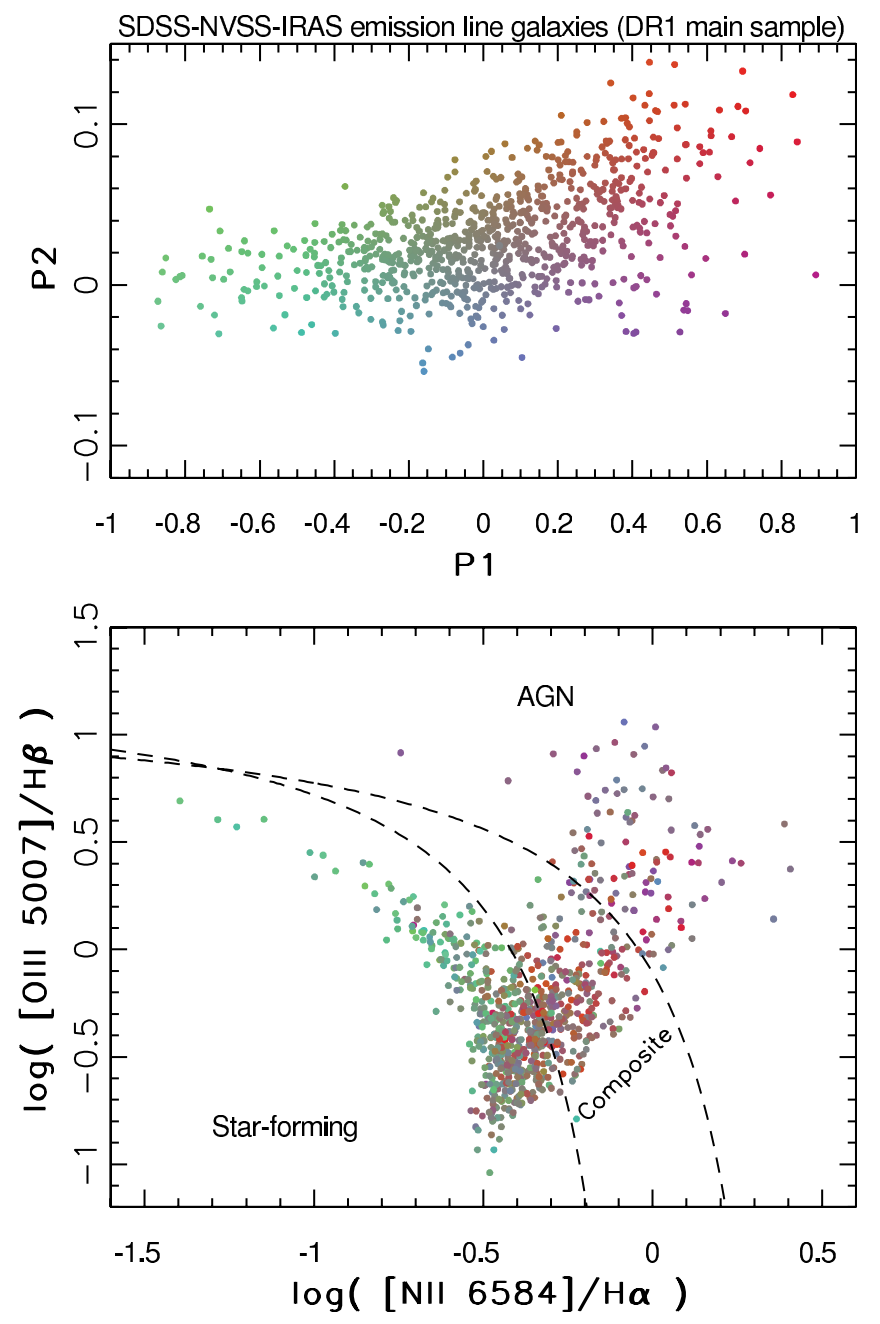

FIG. 25.- Same as Fig. 4, but for $\sim 830$ SDSS/NVSS/IRAS emission-line galaxies.
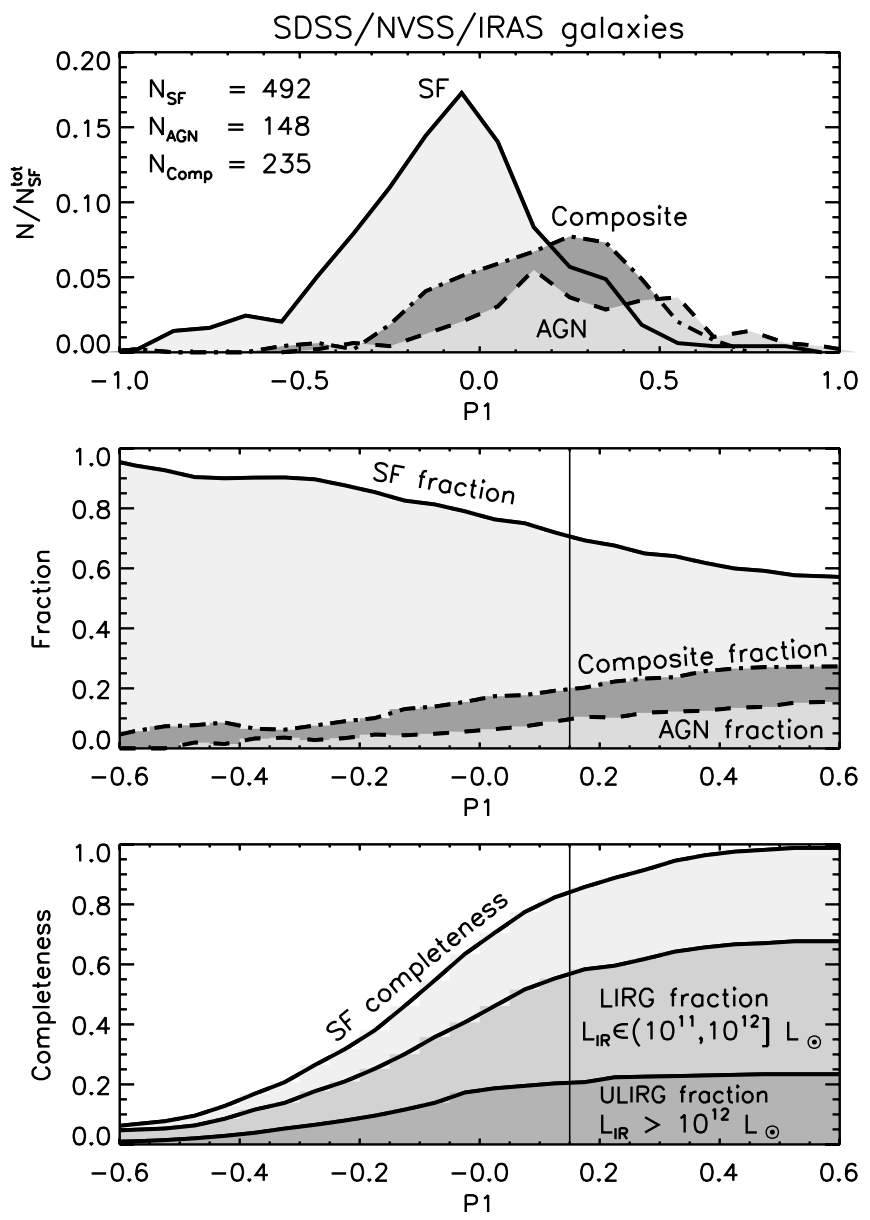

FIG. 26.-Differential and cumulative distributions of the rest-frame color $P 1$ for 875 SDSS galaxies from the DR 1 "main" spectroscopic sample that have NVSS and IRAS detections. The galaxies were spectroscopically classified as SF (SF; thick line), AGN (dashed line) or composite (dot-dashed line; see text for details), and their $P 1$ histograms are shown in the top panel. The middle panel shows the fraction of $P 1$ for $\mathrm{SF}, \mathrm{AGN}$, and composite galaxies, analogous to the middle left panel in Fig. 24. The vertical thin solid line shows the chosen $P 1$ color cutoff. The bottom panel shows the cumulative distribution of $P 1$, normalized to SF galaxy counts (thick line). Also shown are the $P 1$ distributions of SF galaxies with IR luminosities greater than $10^{11}$ and $10^{12} L_{\odot}$ consistent with LIRGs and ULIRGs, respectively. Note that our $z_{\text {spec }}$ rest-frame color-based classification method is not strongly biased against dusty starburst galaxies.

The differential distribution of $P 1$ for the complete sample of 875 SDSS/NVSS/IRAS galaxies is shown in the top panel of Figure 24 . The galaxies were spectroscopically separated into AGN, SF, and composite galaxies (as described in $\S$ 4.1.1). In the middle panel we show the corresponding fraction (analogous to the middle left panel in Fig. 24). Using a rest-frame $P 1$ color cutoff of 0.15 the selected sample of SF galaxies, detected in the IR regime, contains $\sim 70 \%$ real (i.e., spectroscopically identified) SF galaxies (which make up $\sim 85 \%$ of all real SF galaxies; see Fig. 24 , bottom), $10 \%$ AGNs, and $20 \%$ composite objects. This is fairly consistent with the properties of the entire SDSS/NVSS sample.

In order to assess the fraction of dusty starburst galaxies that we omit using the rest-frame color selection, in the bottom panel of Figure 26 we show the cumulative distributions of the spectroscopically identified SF galaxies as a function of $P 1$ for (1) all SF galaxies, (2) luminous IR galaxies (LIRGs; $L_{\mathrm{IR}}=10^{11}-10^{12} L_{\odot}$ ), and (3) ultraluminous IR galaxies (ULIRGs, $L_{\mathrm{IR}}>10^{12} L_{\odot}$ ). (The total IR luminosities were computed following Sanders \& Mirabel [1996].) A $P 1$ cut of 0.15 misses only $\sim 10 \%$ and $\sim 5 \%$ of LIRGs and ULIRGs, respectively.

Afonso, J., Georgakakis, A., Almeida, C., Hopkins, A. M., Cram, L. E., Mobasher, B., \& Sullivan, M. 2005, ApJ, 624, 135

Alexander, D. M., Brandt, W. N., Hornschemeier, A. E., Garmire, G. P.,

Schneider, D. P., Bauer, F. E., \& Griffiths, R. E. 2001, AJ, 122, 2156

Appleton, P. N., et al. 2004, ApJS, 154, 147

Arnouts, S., et al. 2002, MNRAS, 329, 355

Baldwin, J. A., Phillips, M. M., \& Terlevich, R. 1981, PASP, 93, 5

\section{REFERENCES}

Barger, A. J., Cowie, L. L., \& Wang, W.-H. 2007, ApJ, 654, 764

Becker, R. H., White, R. L., \& Helfand, D. J. 1995, ApJ, 450, 559

Beichman, C. A., Neugebauer, G., Habing, H. J., Clegg, P. E., \& Chester, T. J. 1985, IRAS Catalogs and Atlases (Washington: USGPO)

Bell, E. F. 2003, ApJ, 586, 794

Benn, C. R., Rowan-Robinson, M., McMahon, R. G., Broadhurst, T. J., \& Lawrence, A. 1993, MNRAS, 263, 98 
Best, P. N. 2004, MNRAS, 351, 70

Best, P. N., Kauffmann, G., Heckman, T. M., \& Ivezić, Ž. 2005, MNRAS, 362, 9

Brinchmann, J., Charlot, S., White, S. D. M., Tremonti, C., Kauffmann, G., Heckman, T., \& Brinkmann, J. 2004, MNRAS, 351, 1151

Brusa, M., et al. 2007, ApJS, 172, 353

Bruzual, G., \& Charlot, S. 2003, MNRAS, 344, 1000

Bondi, M., Ciliegi, P., Schinnerer, E., Smolčić, V., Jahnke, K., Carilli, C., \& Zamorani, G. 2008, ApJ, in press (arXiv: 0804.1706)

Capak, P., et al. 2007, ApJS, 172, 99

Cappelluti, E., et al. 2007, ApJS, 172, 341

Caputi, K. I., et al. 2007, ApJ, 660, 97

Chan, B. H. P., Cram, L. E., Sadler, E. M., Killeen, N. E. B., Jackson, C. A., Mobasher, B., \& Ekers, R. D. 2004, MNRAS, 352, 1245

Colless, M., et al. 2001, MNRAS, 328, 1039

Condon, J. J. 1984, ApJ, 287, 461 1992, ARA\&A, 30, 575

Condon, J. J., Cotton, W. D., Greisen, E. W., Yin, Q. F., Perley, R. A., Taylor, G. B., \& Broderick, J. J. 1998, AJ, 115, 1693

Cowie, L. L., Barger, A. J., Fomalont, E. B., \& Capak, P. 2004, ApJ, 603, L69

Daddi, E., Cimatti, A., Renzini, A., Fontana, A., Mignoli, M., Pozzetti, L., Tozzi, P., \& Zamorani, G. 2004, ApJ, 617, 746

Fanaroff, B. L., \& Riley, J. M. 1974, MNRAS, 167, 31P

Finoguenov, A., et al. 2007, ApJS, 172, 182

Fomalont, E. B., Kellermann, K. I., Cowie, L. L., Capak, P., Barger, A. J., Partridge, R. B., Windhorst, R. A., \& Richards, E. A. 2006, ApJS, 167, 103

Franzetti, P. 2005, Ph.D. thesis, Universita' di Milano-Bicocca

Georgakakis, A., Mobasher, B., Cram, L., Hopkins, A., Lidman, C., \& RowanRobinson, M. 1999, MNRAS, 306, 708

Gruppioni, C., Mignoli, M., \& Zamorani, G. 1999, MNRAS, 304, 199

Guzzo, L., et al. 2007, ApJS, 172, 254

Haarsma, D. B., Partridge, R. B., Windhorst, R. A., \& Richards, E. A. 2000, ApJ, 544, 641

Hasinger, M., et al. 2007, ApJS, 172, 29

Hauschildt, P. H., Baron, E., \& Allard, F. 1997, ApJ, 483, 390

Hopkins, A. M. 2004, ApJ, 615, 209

Hopkins, A. M., et al. 2003, ApJ, 599, 971

Huynh, M. T., Jackson, C. A., Norris, R. P., \& Prandoni, I. 2005, AJ, 130, 1373

Ilbert, O., et al. 2006, A\&A, 457, 841

Impey, C. D., et al. 2007, in IAU Symp. 238, A Survey of AGN and Supermassive Black Holes in the COSMOS Survey, ed. V. Karas \& G. Matt (Cambridge: Cambridge Univ. Press), 287

Ivezić, Ž., et al. 2002, AJ, 124, 2364

Jackson, C. A., \& Londish, D. M. 2000, Publ. Astron. Soc. Australia, 17, 234

Jarvis, M. J., \& Rawlings, S. 2004, NewA Rev., 48, 1173

Kauffmann, G., et al. 2003, MNRAS, 346, 1055

Kewley, L. J., Dopita, M. A., Sutherland, R. S., Heisler, C. A., \& Trevena, J. 2001, ApJ, 556, 121

Koekemoer, E., et al. 2007, ApJS, 172, 196

Kong, X., Charlot, S., Brinchmann, J., \& Fall, S. M. 2004, MNRAS, 349, 769

Lacy, M., et al. 2004, ApJS, 154, 166
Leauthaud, A., et al. 2007, ApJS, 172, 219

Le Floc'h, E., et al. 2005, ApJ, 632, 169

Lilly, E., et al. 2007, ApJS, 172, 70

Maccacaro, T., Gioia, I. M., Wolter, A., Zamorani, G., \& Stocke, J. T. 1988, ApJ, 326, 680

Madau, P., Ferguson, H. C., Dickinson, M. E., Giavalisco, M., Steidel, C. C., \& Fruchter, A. 1996, MNRAS, 283, 1388

Obrić, M., et al. 2006, MNRAS, 370, 1677

Odell, A. P., Schombert, J., \& Rakos, K. 2002, AJ, 124, 3061

Padovani, P., Mainieri, V., Tozzi, P., Kellermann, K. I., Fomalont, E. B., Miller, N., Rosati, P., \& Shaver, P. 2007, in ASP Conf. Ser. 380, At the Edge of the Universe, ed. J. Afonso et al. (San Francisco: ASP), 205

Peterson, B. M. 1997, An Introduction to Active Galactic Nuclei (New York Cambridge Univ. Press)

Polletta, M. d. C., et al. 2007, ApJ, 663, 81

Prescott, M. K. M., Impey, C. D., Cool, R. J., \& Scoville, N. Z. 2006, ApJ, 644, 100

Richards, G. T., et al. 2002, AJ, 123, 2945

Rola, C. S., Terlevich, E., \& Terlevich, R. J. 1997, MNRAS, 289, 419

Sadler, E. M., McIntyre, V. J., Jackson, C. A., \& Cannon, R. D. 1999, Publ. Astron. Soc. Australia, 16, 247

Salim et al. 2007, ApJS, 173, 267

Sanders, D. B., \& Mirabel, I. F. 1996, ARA\&A, 34, 749

Sanders, E., et al. 2007, ApJS, 172, 86

Seymour, N., McHardy, I. M., \& Gunn, K. F. 2004, MNRAS, 352, 131

Schinnerer, E., et al. 2007, ApJS, 172, 46

Schneider, D. P., et al. 2005, AJ, 130, 367

Scoville, E., et al. 2007a, ApJS, 172, 38

Scoville, N., et al. 2007b, ApJS, 172, 150

Silva, L., Granato, G. L., Bressan, A., \& Danese, L. 1998, ApJ, 509, 103

Simpson, C., et al. 2006, MNRAS, 372, 741

Smolčić, V., et al. 2004, ApJ, 615, L141

2006, MNRAS, 371, 121

2007b, ApJS, 172, 295

2008, ApJ, submitted

Stern, D., et al. 2005, ApJ, 631, 163

Strateva, I., et al. 2001, AJ, 122, 1861

Strauss, M. A., et al. 2002, AJ, 124, 1810

Szkody, P., et al. 2002, AJ, 123, 430 2003, AJ, 126, 1499

Taniguchi, Y., et al. 2007, ApJS, 172, 9

Tran, Q. D., et al. 2001, ApJ, 552, 527

Trump, J. R., et al. 2007, ApJS, 172, 383

Veilleux, S., \& Osterbrock, D. E. 1987, ApJS, 63, 295

Veilleux, S., Sanders, D. B., \& Kim, D.-C. 1999, ApJ, 522, 139

Willott, C. J., Rawlings, S., Blundell, K. M., Lacy, M., Hill, G. J., \& Scott, S. E. 2002, MNRAS, 335, 1120

Windhorst, R. A., Miley, G. K., Owen, F. N., Kron, R. G., \& Koo, D. C. 1985, ApJ, 289, 494

York, D. G., et al. 2000, AJ, 120, 1579 